\title{
Noninvasive emittance and energy spread monitor using optical synchrotron radiation
}

\author{
R. Fiorito, ${ }^{1,2, *}$ A. Shkvarunets, ${ }^{1}$ D. Castronovo, ${ }^{3}$ M. Cornacchia ${ }^{3}$ S. Di Mitri, ${ }^{3}$ \\ R. Kishek, ${ }^{1}$ C. Tschalaer, ${ }^{4}$ and M. Veronese ${ }^{3}$ \\ ${ }^{1}$ University of Maryland, College Park, Maryland 20742, USA \\ ${ }^{2}$ Cockcroft Institute, University of Liverpool, Daresbury WA4 4AD, United Kingdom \\ ${ }^{3}$ Elettra-Sincrotrone Trieste S.C.p.A., Basovizza 34149, Italy \\ ${ }^{4}$ Massachusetts Institute of Technology, Cambridge, Massachusetts 02139, USA \\ (Received 20 March 2014; published 19 December 2014)
}

\begin{abstract}
We propose a design for a minimally perturbing diagnostic minichicane, which utilizes optical synchrotron radiation (OSR) generated from magnetic bends in the chicane, to measure the rms horizontal and vertical beam sizes, divergences, emittances, Twiss parameters and energy spread of a relativistic electron beam. The beam is externally focused to a waist at the first bend and the OSR generated there can be used to measure the rms beam size. Subsequent pairs of bends produce far field OSR interferences (OSRI) whose visibility depends on the beam energy spread and the angular divergence. Under proper conditions, one of these two effects will dominate the OSRI visibility from a particular pair of bends and can be used to diagnose the dominant effect. The properties of different configuration of bends in the chicane have been analyzed to provide an optimum diagnostic design for a given set of beam parameters to: (1) provide a sufficient number of OSR interferences to allow a measurement of the fringe visibility; (2) minimize the effect of coherent synchrotron radiation and space charge forces on the particles motion; and (3) minimize the effect of compression on the bunch length as the beam passes through the chicane. A design for the chicane has been produced for application to the FERMI free electron laser facility and by extension to similar high brightness linear accelerators. Such a diagnostic promises to greatly improve control of the electron beam optics with a noninvasive measurement of beam parameters and allow on-line optics matching and feedback.
\end{abstract}

DOI: 10.1103/PhysRevSTAB.17.122803

PACS numbers: 41.60.-m, 41.75.Ht, 42.79.-e

\section{INTRODUCTION}

Optical synchrotron radiation (OSR) and optical edge radiation interferences from preexisting magnetic bends and field transitions in storage rings and linacs have previously been studied and utilized to measure beam parameters [1-3]. However, the theory and experiments to measure these parameters depend on observations of OSR in the Fresnel zone where beam and divergence effects are not well separated. In contrast to previous papers we propose an approach that can easily distinguish between the beam's spatial and angular properties. This is accomplished by simultaneous observations of the source distribution of OSR and the far field OSR interference (OSRI) patterns from two sources, to independently obtain the beam size and divergence, respectively [4]. This approach, which is similar to the one we have successfully employed using OTR and OTRI [5-8], is simple to set up experimentally

\footnotetext{
*Corresponding author. ralph.fiorito@cockcroft.ac.uk
}

Published by the American Physical Society under the terms of the Creative Commons Attribution 3.0 License. Further distribution of this work must maintain attribution to the author $(s)$ and the published article's title, journal citation, and DOI. and can easily separate out size and divergence effects, which are interwoven in the Fresnel approach.

Our primary goal in this paper is to present a benchmark design for a compact diagnostic chicane based on observations of OSR that (1) can measure both horizontal and vertical transverse normalized emittances $\sim 1$ micron, which are typically produced by state-of-the-art photoinjectors; (2) is noninvasive, i.e., does not significantly alter the beam's fundamental properties, in particular the transverse emittance and bunch length, during the course of the measurements; (3) is optimized for a given set of operating beam conditions typically used at free electron lasers (FELs); (4) can additionally measure energy spread; and (5) can serve as the basis of a slow feedback system to adjust the accelerator to transport variations and maintain the beam brightness.

We present a design for a minichicane that is matched to the beam line via external quadrupole magnets. The beam line optics is intended to be suitable for diagnostic purposes as well as for beam production. The chicane uses four small electromagnets to perturb the beam trajectories just enough to produce OSR and OSRI, which can be used to diagnose the beam parameters, and then return the beam to its original trajectory on axis. To design the chicane we use the 
ELEGANT [9] and WARP [10] simulation codes as well as a special OSR code that we have developed [4] to calculate the far field angular distribution of synchrotron radiation from an electron moving along an arbitrary trajectory. This code has been extensively checked against data and output from other synchrotron codes and is able to calculate the effect of beam energy spread and angular divergence on OSRI generated from any pair of magnetic dipoles.

The structure of this paper is as follows. Section II gives an overview of the properties of OSR interferences generated from chicane bends and some background on the code we have developed to calculate OSRI for various magnetic trajectories. Section III presents the general design for a noninvasive energy spread and emittance diagnostic chicane (NIEM) and our strategy to measure the beam emittance and energy spread with the NIEM. Section IV gives a description of the FERMI accelerator system and requirements on the NIEM for use as an online monitor in the FERMI FEL linac. In Sec. V we discuss the beam properties of FERMI, calculated using the ELEGANT and WARP simulation codes at two positions and energies (100 and $286 \mathrm{MeV})$ in the transport system, where NIEMs would be useful as online monitors. Our OSRI code results are presented in Sec. VI, which indicate how the NIEM can be used to measure energy spread and emittance at the candidate energies. Some experimental considerations needed to measure the required beam parameters are given in Sec. VII. Section VIII explains how NIEM can be used in a feedback loop to control and maintain the electron beam brightness. Finally, in Sec. IX we present our conclusions. In addition, two Appendices are included: Appendix A provides details on how the beam parameters vary as they pass through the NIEM chicane and Appendix B describes a simplified, approximate analysis of the effect of beam parameters on OSR interferences, based on the analogy to OTR interferences, which in lieu of employing a full OSRI simulation code, can be utilized to evaluate a NIEM for other accelerators.

\section{OSR INTERFEROMETRY}

\section{A. Magnetic geometries}

Chicanes typically have pairs of magnets arranged in both "S" and "U" geometries. The features of the synchrotron radiation from each bend and the consequent overlap of the radiation patterns generated in these two geometries are depicted schematically in Fig. 1.

Figure 1 shows the far field OSR radiation patterns (shown as grey and white ovals) generated by an electron traveling along the trajectory for different pairs of magnetic bends. Note that for the $\mathrm{U}$ configuration the radiation patterns (represented by the two grey ovals) overlap and thus interfere only in a very limited angular region $\left(\Delta \theta \approx \gamma^{-1}\right)$, where $\gamma$ is the beam's relativistic Lorentz factor. In contrast, for an $\mathrm{S}$ configuration the angular distribution patterns of SR generated at the two bends

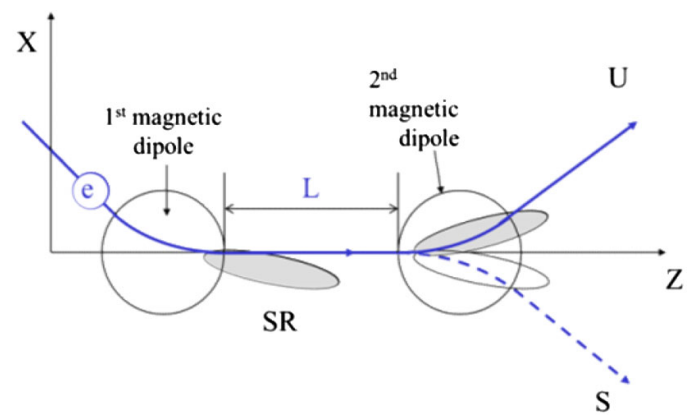

FIG. 1. Geometries for generation of synchrotron radiation from two magnetic dipoles in a $\mathrm{U}$ or an $\mathrm{S}$ configuration.

(grey and white ovals) completely overlap. In the latter case the angular region of interference and the peak intensity of the OSRI is much larger than in the $\mathrm{U}$ case.

\section{B. Properties of the OSR interferences}

The OSR interferences are generated by the coherent addition of the radiation fields of photons moving in the same direction and generated by the same electron, while it moves along the curved trajectory. The relativistic electron radiates forward in the direction tangential to the curved trajectory at the moment when the electron passes this elemental arc. In the case of a complex trajectory (see Fig. 1), there are elemental arcs (sources) in the trajectory that are separated in space and radiate overlapping photons which propagate in the same direction. These photons have a phase difference that depends on the distance between the two sources of radiation, the electron's energy and the trajectory between the sources. For each pair of elemental SR radiators with overlapping radiation patterns the situation is similar to optical transition radiation generated by an electron transiting two foils, which produce OTR photons at each foil and consequently OTR interferences (OTRI) [5]. In this paper we assume that the electrons in a bunch radiate incoherently, a model that is applicable when the distribution of electrons is "smooth" and has a width that is much larger than the wavelength of radiation. We therefore assume that there is negligible microbunching at optical wavelengths (cf. Sec. V); thus, the total OSRI intensity from the bunch is the sum of the intensities of the OSR interferences from all the electrons and is proportional to the number of electrons.

In general, each electron in the bunch moving in the transverse magnetic field follows a different trajectory either due to a difference in magnetic deflection produced if the electron has a different energy, or because it has a different transverse momentum than its neighbors. Hence the electrons will generate different interference patterns with different fringe structures (i.e., the angular position of maxima and minima). The total interference pattern of radiation produced by the incoherent ensemble of particles can be found by following the path of each particle, 


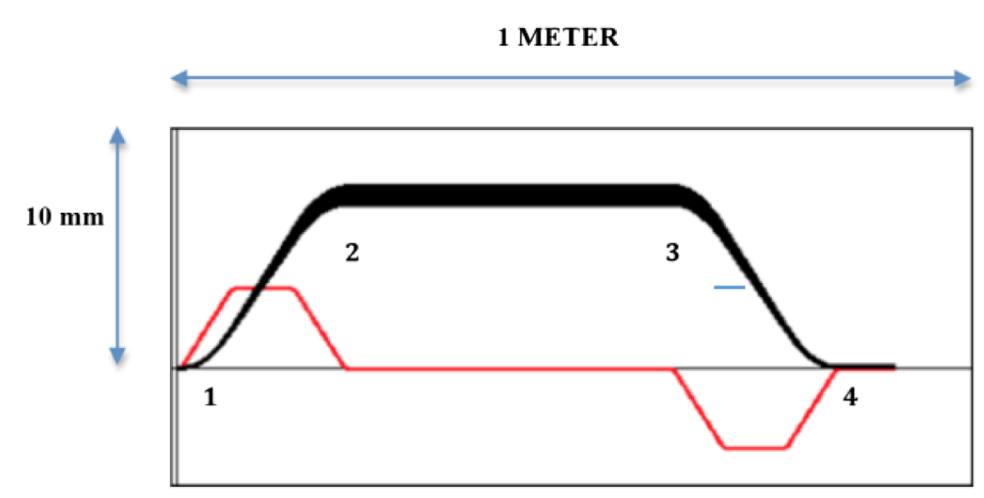

FIG. 2. Schematic (not to scale) of diagnostic chicane showing electron trajectories (black) and deflection angles (red).

computing the OSRI for that trajectory and then incoherently adding the OSRI intensity patterns from all the electrons in the bunch. The visibility of fringes (the depth of modulation) of this summed OSRI pattern will depend on the energy spread-induced angular spread of the trajectories, if applicable, the inherent energy spread of the particles and the beam's intrinsic (i.e. betatron) angular divergence induced by the spread in transverse momenta of the particles. However, it is possible that the visibility of the OSRI fringes produced from a particular pair of magnets will be dominated by one of these parameters and thus can be used to measure that parameter. We will demonstrate this with a chicane that utilizes OSR interferences from two different pairs of dipoles, to measure the energy spread and divergence of the electron beam of the FERMI FEL accelerator [11].

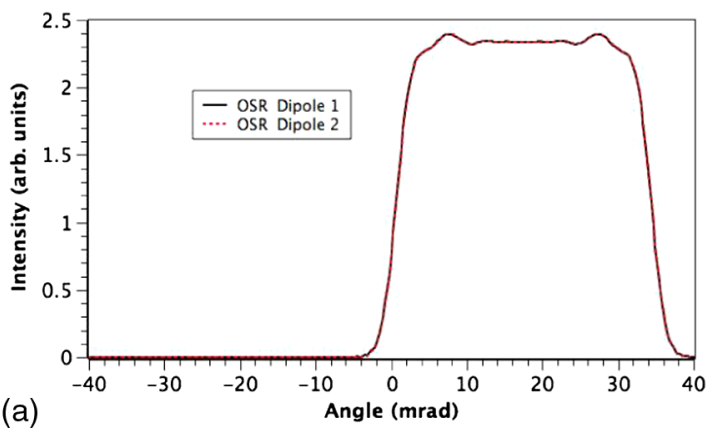

\section{NIEM chicane design}

A sketch of the chicane that we propose as a nonintercepting beam emittance and energy spread monitor (NIEM) is shown in Fig. 2. The parameters of this chicane are specific to FERMI. However, the general principles underlying the design and its application to beam diagnostics are universal and can be applied to other accelerators. We will first give an overview of the design and its diagnostic properties. Details of the design will be presented later in the paper.

Figure 2 shows the trajectories (black lines) and average bending angles $(+35$ to $-35 \mathrm{mrad}$; red line) of the beam electrons as they pass through the chicane. The first two magnetic dipoles $(1,2)$ of the chicane form an $\mathrm{S}$ type interferometer that will be used to monitor the energy spread. The second and third dipoles $(2,3)$, which form an

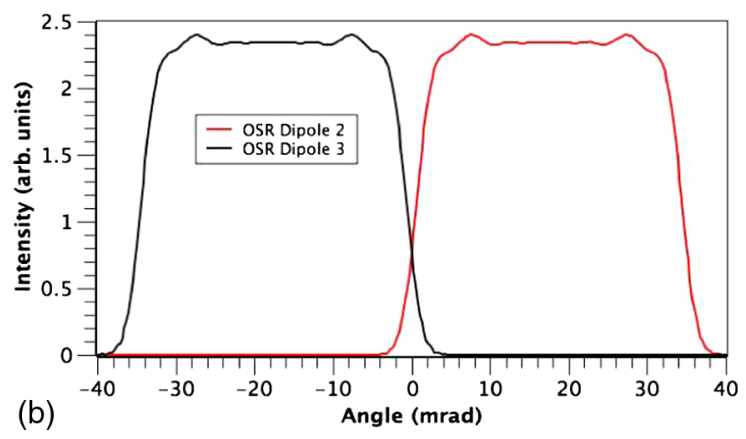

(b)

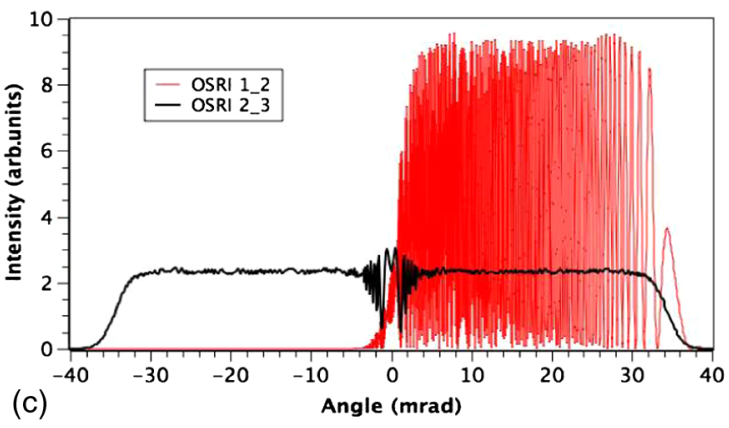

(c)

FIG. 3. Far field angular distribution patterns of OSR generated from a $100 \mathrm{MeV}$ electron passing through two dipoles arranged: (a) in an $\mathrm{S}$ configuration (dipoles 1,2); (b) in a $\mathrm{U}$ configuration (dipoles 2,3); and (c) corresponding $\mathrm{S}$ and $\mathrm{U}$ interference patterns. 
inverted U interferometer, will be used to measure the beam divergence. Dipole pairs 3,4 also form an S configuration similar to $(1,2)$ that can also be utilized for diagnostics, when convenient, but they are not discussed here. The chicane is designed to minimally perturb the FERMI beam but still produce sufficient OSR and OSRI to diagnose the beam parameters.

Figure 3(a) shows the overlapping OSR patterns generated from dipoles 1 and 2 for a $100 \mathrm{MeV}$ beam; Fig. 3(b) shows similar patterns from dipoles 2 and 3; Fig. 3(c) shows the resulting interference patterns from each dipole pair. The $\mathrm{S}$ configuration from magnets 1,2 produces an interference pattern over the entire angular region subtended by the bend, with $100 \%$ modulation, if the visibility is not altered by the presence of energy spread, divergence or optical band pass. For the $\mathrm{U}$ configuration from magnets 2,3 the situation is different, i.e., the interferences are observable only from the edges of the distribution, the fringe fields of the magnets, and the modulation depth is much more shallow over the region of interference. In all cases the initial trajectory designates zero degrees, and positive angles are measured in the counterclockwise direction. The shapes of the OSR distributions depend on beam and field parameters, which we will elucidate later. However, the overlap and resulting gross features of the OSRI patterns for all $\mathrm{U}$ and $\mathrm{S}$ configurations will be similar.

\section{DIAGNOSTIC MEASUREMENT SYSTEM}

\section{A. Emittance measurement}

The electron beam rms emittance can be determined in several ways. The method we will concentrate on in this paper is to magnetically focus the beam to a waist condition at the entrance of the first bend of the chicane using quadrupoles installed upstream of the chicane. A detailed explanation on how this will be accomplished is presented in Sec. VIII. An optical imaging system focused on the OSR from the first bend can be used to observe and measure the beam's transverse dimensions. Two other imaging systems that are focused at infinity independently observe the far field radiation OSR interference patterns from bends 1,2 and 2,3. The visibilities of the interferences from these bend pairs are used to obtain the beam energy spread and the betatron beam divergence, respectively. Since the betratron divergence is constant throughout the chicane and the correlation between the particles' positions and angles is zero at a beam waist (e.g., $\left\langle x x^{\prime}\right\rangle=0$ ), the product of the rms beam size and divergence gives the local emittance. Also, we will show that the horizontal $(x)$ and vertical $(y)$ components of the rms beam size and divergence are decoupled and therefore can both be simultaneously measured. With this approach the emittance and energy spread can be monitored continuously, which is the goal of the application of the NIEM at FERMI. We also note that the electron beam optics design for NIEM is fully compatible with the beam transport for FEL production at FERMI and can be similarly used at other high brightness accelerators.

\section{B. Optics setup}

A schematic of the optical arrangement for the NIEM diagnostics monitor for FERMI is shown in Fig. 4. The optics below the chicane observe the OSR from the beam entering the first dipole. In the sketch shown in Fig. 4, the OSR from the first dipole passes through a Young's double slit. With this arrangement, the beam size can be determined by measurement of the visibility of the resulting far field diffraction pattern, observed by the bottom left by "Camera 1." The visibility of the diffraction fringes is a function of the beam size and can be used to measure sizes down to a few microns $[12,13]$. However, as is discussed later in Sec. VIII, if the beam size is a few times larger than the point spread function (PSF) of the optics system, one can also use direct imaging to measure the beam size. In this case the double slit and bandpass filter can be removed and the camera can be focused directly on the entrance of dipole 1. Or, if desired, a beam splitter and an additional camera can be installed in the optics to enable both types of beam size measurements.

"Camera 1,2" shown in the top left part of Fig. 4 views the far field OSRI pattern from bends 1,2; that are observed in the horizontal $(x)$ plane. As we will show below for the beam parameters of interest at FERMI, these interferences are primarily sensitive to the energy spread of the beam. Lastly, "Camera 2,3" shown in the upper right of the figure observes the far field OSRI pattern from bends 2,3, whose $(x, y)$ visibilities are chiefly determined by the $(x, y)$ betatron beam divergences. All three systems require and include narrow bandpass filters (e.g., $10 \mathrm{~nm}$ at $630 \mathrm{~nm}$ for

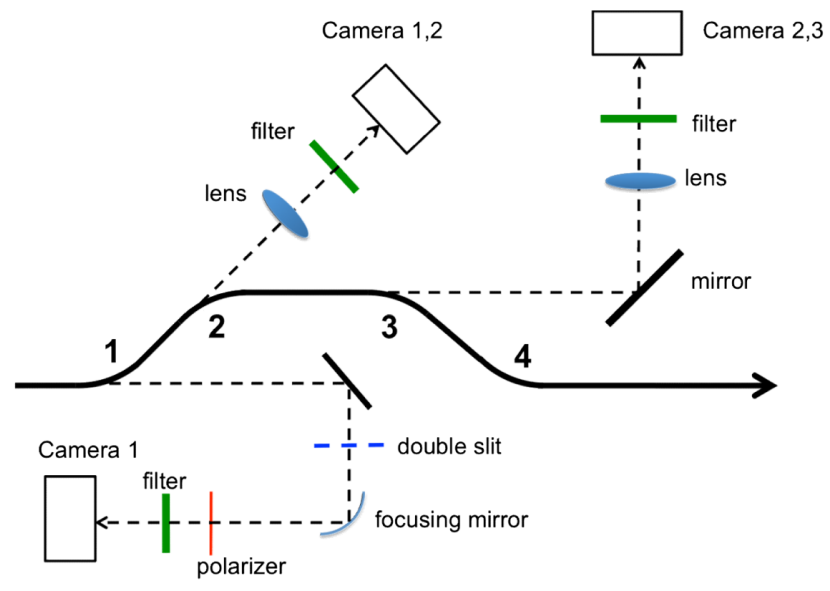

FIG. 4. Optics setup for measurement of (a) beam size, (b) energy spread, and (c) betatron divergence via observations of (a) OSR from bend 1; (b) OSRI from bends 1,2; and (c) OSRI from bends 2,3 , respectively. 
TABLE I. FERMI FEL main operating parameters.

\begin{tabular}{lccc}
\hline \hline Parameter & FEL-1 & FEL-2 & Unit \\
\hline Charge & $500-800$ & $500-800$ & $\mathrm{pC}$ \\
Energy & $0.9-1.2$ & $1.1-1.5$ & $\mathrm{GeV}$ \\
Peak current & $<800$ & $<800$ & $\mathrm{~A}$ \\
$\begin{array}{l}\text { Final bunch length, FWHM } \\
\quad \text { full width half maximum) }\end{array}$ & $>0.5$ & 0.7 & pico seconds \\
Normalized emittance rms, & 1.5 & $<1.5$ & $\mu \mathrm{m}$ \\
$\quad$ slice & & & \\
Energy spread rms, slice & $<250$ & $<250$ & $\mathrm{keV}$ \\
$\begin{array}{l}\text { Compression factor } \\
\text { Fundamental wavelength }\end{array}$ & $100-20$ & $20-4$ & $\mathrm{nano} \mathrm{meters}$ \\
& & & $(\mathrm{nm})$ \\
Energy per pulse & $<400$ & $<100$ & $\mu \mathrm{J}$ \\
Repetition rate & $10 / 50$ & $10 / 50$ & $\mathrm{~Hz}$ \\
\hline \hline
\end{tabular}

Cameras 1,2 and 2,3) to minimize the additional reduction of the fringe visibilities due to wavelength dispersion, and all cameras are focused to infinity, i.e., the sensors of these cameras are positioned at the focal planes of their respective focusing elements.

\section{FERMI FEL PARAMETERS}

FERMI is a single-pass fourth generation light source user facility in operation at Elettra Sincrotrone Trieste in Italy. Table I shows the main electron and photon beam parameters. An electron beam in the energy range 0.9-1.5 GeV drives two seeded FELs in the fundamental wavelength range 4-100 $\mathrm{nm}[14,15]$. The accelerator and FEL complex comprise the following parts: a normal conducting, S-band photoinjector and a main linac in which the beam is time compressed in one or two stages by a total factor of $\sim 10$; the transport system to the undulators; the undulator complex where the FEL radiation is generated; the photon beam lines, which transport the radiation from the undulator to the experimental area; and the experimental area itself.

We have designed chicanes that can be used to monitor the emittance at two different locations of interest in the FERMI beam line, which is sketched in Fig. 5. The layouts are compatible with available space to insert the NIEM and the electron optics to match the beam to the NIEM using the current operating Twiss parameters. The first location is upstream of the laser heater (LH in Fig. 5) system [16] that is run to suppress microbunching instability, and thus maximize the FEL performance [17]. The beam energy here is $100 \mathrm{MeV}$. This point is interesting because from this energy onward transverse space charge effects are negligible and, in absence of other perturbations (e.g., coherent synchrotron radiation, linac geometric wakefields, chromatic effects, etc., all of which are minimized as much as possible in the FERMI design), the normalized emittances should remain constant. This is also the point before bunch compression, where the bunch is long and the correlated and uncorrelated energy spread are small. Because of these two situations one expects little disruption to the bunch from the NIEM.

A second location of interest for a NIEM device is after the first magnetic chicane for bunch length compression (BC1 in Fig. 5) where the beam energy is $286 \mathrm{MeV}$. This is an interesting region because a NIEM here can observe any possible emittance blowup and optics matching disruption that might have occurred in the compressor chicane, primarily due to coherent synchrotron radiation (CSR). This location potentially presents a more delicate beam dynamics situation, since the bunch will traverse the NIEM with the large correlated energy spread $(\leq 2 \%)$ that is required by the preceding bunch length compression.

The parameters of the NIEM chicanes for the two aforementioned locations are: (1) a magnetic field strength, $B=0.12 \mathrm{~T}$ for $100 \mathrm{MeV}$ and $0.35 \mathrm{~T}$ for $286 \mathrm{MeV}$; (2) dipole magnetic length, $l=100 \mathrm{~mm}$ and $35 \mathrm{mrad}$ bending angle; spacing between magnets $(1,2), L_{12}=$ $100 \mathrm{~mm}$ and $(2,3), L_{23}=600 \mathrm{~mm}$. These parameters are required to easily measure both the rms divergences and beam sizes for normalized emittances in the range of 1 to several microns. The physical specifications of the dipole magnets for the $\mathrm{BC} 1$ area (not shown) allow the desired bending angle with sufficiently small random and systematic magnetic errors in order not to affect the electron beam quality. In spite of the relatively low energy range in the places that they will be used and their compactness, the specified field quality is relaxed with respect to standard dipoles for magnetic compressors [18]. Similar specifications apply, with some relaxed magnetic field components and uniformity, to the LH area. Here, in spite of a lower beam rigidity (i.e., lower beam energy), the energy spread is an order of magnitude smaller than in $\mathrm{BC} 1$, thus relaxing the overall magnetic design for the same bending angle.

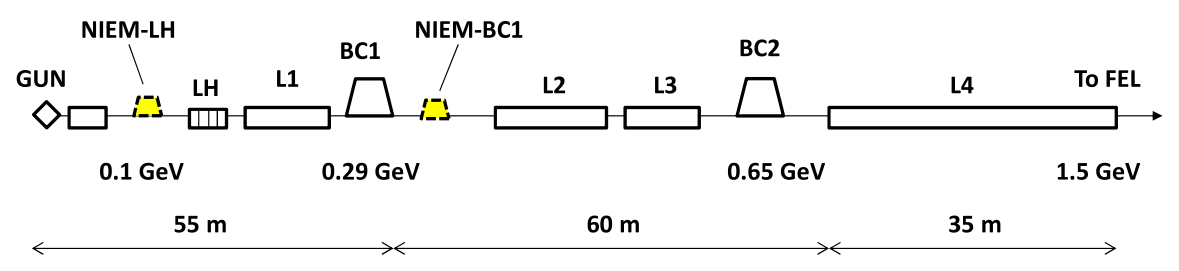

FIG. 5. Sketch of the FERMI beam accelerator complex (not to scale). Possible locations for the NIEM minichicane are shown in the laser heater $(\mathrm{LH})$ and first magnetic compressor (BC1) area. 


\section{ELECTRON BEAM DYNAMICS}

\section{A. Results at 100 and $286 \mathrm{MeV}$}

The NIEM chicane has the physical configuration of a magnetic bunch length compressor and, in principle, is subject to the same potential threats to the electron beam brightness, i.e., (1) emission of CSR and the associated increased projected emittance in the bending plane and increased energy spread; (2) enhancement of microbunching instability driven by longitudinal space charge (LSC); and (3) direct space charge effects at energies $\leq 100 \mathrm{MeV}$. In addition, it is required that the NIEM chicane be as "transparent" as possible to the beam; i.e., contrary to a bunch compressor chicane, it must not change the bunch length at its exit nor enlarge the horizontal beam size and divergence in the radiation monitoring angle beyond a given tolerance, in order for NIEM to measure the "geometric" emittance (i.e., without a contribution from the energy spread).

To study the effects listed above we have employed the ELEGANT and WARP simulation codes. We now summarize below the results of simulations, supported by theoretical considerations. Doing this, we include the effect of CSR and 3rd order transport matrix across the chicane. Figure 6 shows the betatron functions and energy dispersion function across NIEM at $100 \mathrm{MeV}$. Six quadrupoles located a few meters upstream match the Twiss parameters of the beam coming from the injector to those at the NIEM location. Such an optics matching procedure is routinely carried out at FERMI for machine tuning and was adopted to build up the design optics shown in Fig. 6 and following. The agreement between the ELEGANT predictions and the measured values of the Twiss parameters at FERMI is well established [19]. The NIEM leaves the $0.5 \mathrm{nC}, 2.8 \mathrm{ps} \mathrm{rms}$ long bunch's characteristics, that is transverse emittances, bunch length and energy spread totally unchanged.

Another candidate position for NIEM is just past $\mathrm{BC} 1$. Unlike the previous $100 \mathrm{MeV}$ case, here the bunch possesses a rather large $\leq 2 \% \mathrm{rms}$ correlated energy

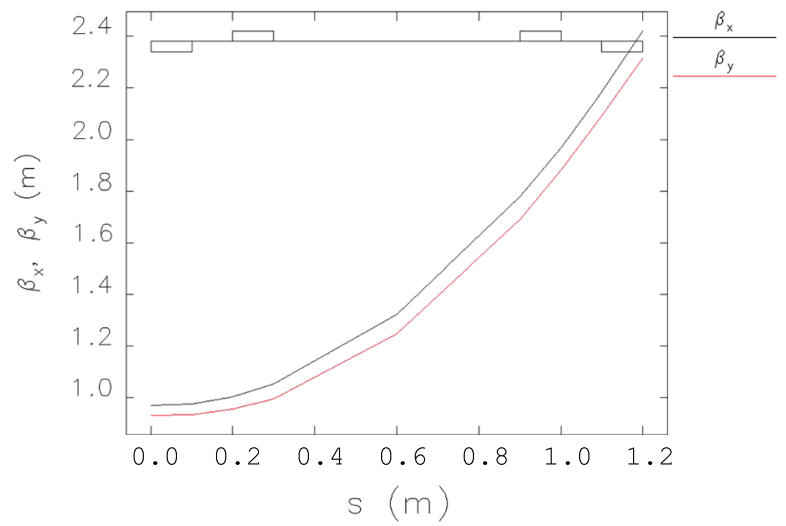

spread $(\delta)$ set up by the preceding linac section, which is required to magnetically compress the bunch. This fact raises the concern that NIEM might also act as a bunch compressor. To avoid this, the NIEM design has small momentum compaction $\left(R_{56}=-0.4 \mathrm{~mm}\right)$ so that the bunch length variation $\Delta z \cong R_{56} \delta$ is negligible with respect to the incoming bunch length. The second order momentum compaction $\left(T_{566}\right)$ does not play a role either, since $T_{566} \delta^{2} \approx O\left(R_{56} \delta^{2}\right) \ll R_{56} \delta$. Figure 7 shows the design optics and energy dispersion functions through the NIEM at $286 \mathrm{MeV}$. Like at $100 \mathrm{MeV}$, the beam properties hardly change: the transverse emittance grows by $4 \%$ and $1 \%$ in the $x$ and the $y$ plane, respectively; the bunch length reduces by $5 \%$.

\section{B. Space charge forces}

The effect of space charge forces on the transverse beam emittance at $100 \mathrm{MeV}$ has been estimated using the WARP simulation code. The results show that space charge at a peak beam current of $130 \mathrm{~A}$ or less will have a negligible effect on the emittance; and, furthermore, that one has to go up to $1 \mathrm{kA}$ peak current ( $1 \mathrm{nC}$ in $1 \mathrm{ps}$ ) to see appreciable space charge effects, i.e., a $1 \%$ change in the emittance and a $26 \%$ change in the beam radius. We recall that the peak current in the FERMI case is approximately $50 \mathrm{~A}$. Moreover, theory [20] predicts that the maximum value of the LSC-driven microbunching spectral gain due to the NIEM only is of the order of unity (1D linear model, analytical), i.e., there will be no further microbunching instability amplification in the NIEM-LH. Similar considerations hold at $286 \mathrm{MeV}$, where the higher beam energy in fact suppresses any space charge effect in spite of the higher bunch peak current, up to approximately $500 \mathrm{~A}$.

\section{Coherent synchrotron radiation}

CSR may be a real threat to the preservation of electron beam transverse emittance and Twiss functions. Since CSR-induced transverse emittance growth, in the bending

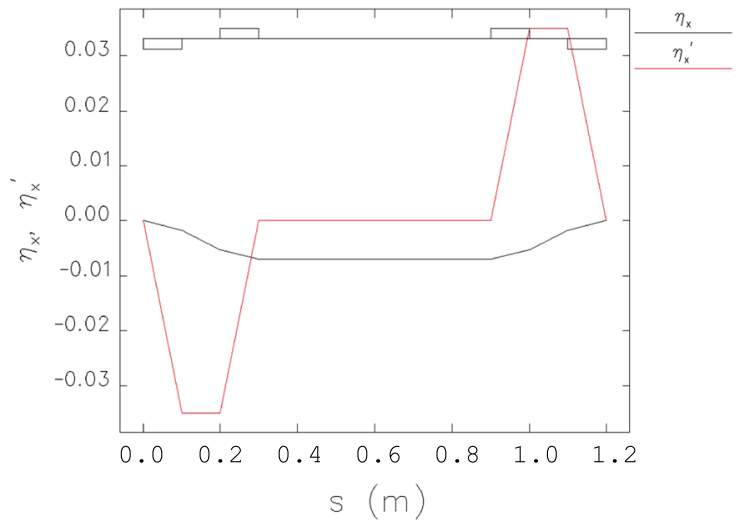

FIG. 6. Betatron functions (left), horizontal energy dispersion function and their s derivatives (right) calculated along NIEM at $100 \mathrm{MeV}$. 

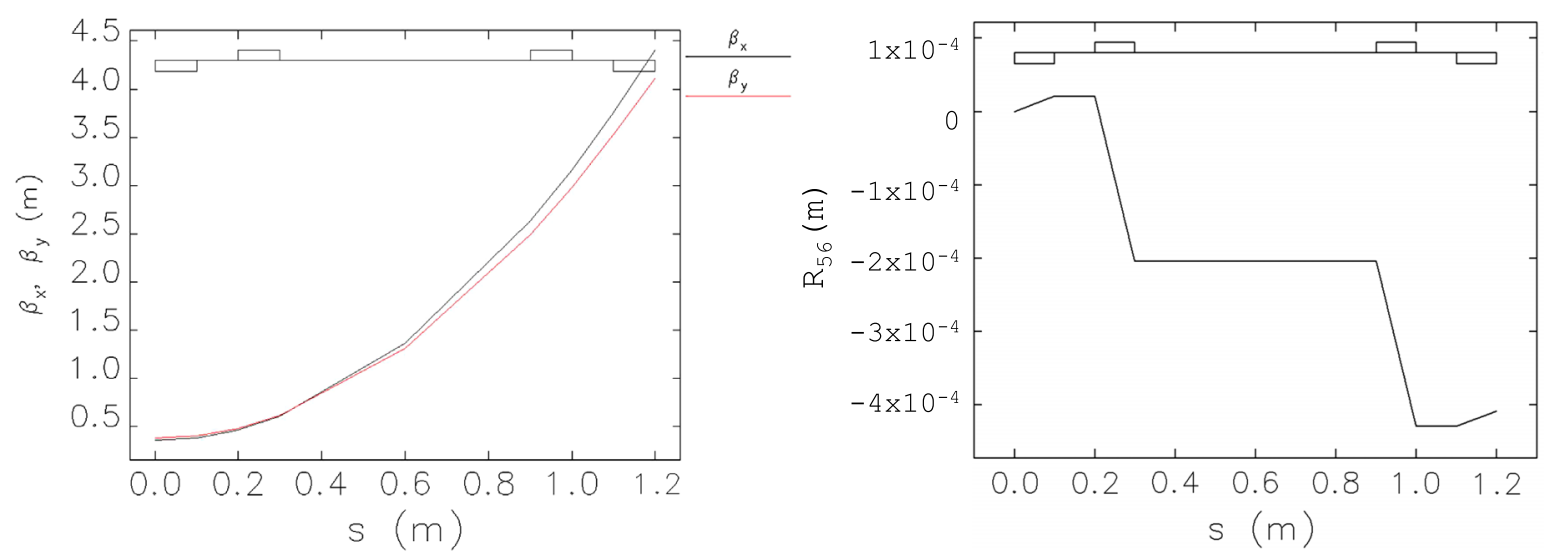

FIG. 7. Betatron functions (left) and $R_{56}$ transport matrix term (right) across NIEM at $286 \mathrm{MeV}$.

plane, is proportional to the CSR-induced energy spread relative to the beam mean energy and inversely proportional to the bunch length, CSR could be particularly harmful in the NIEM at $100 \mathrm{MeV}$, because of the relatively low energy and at $286 \mathrm{MeV}$, because of the bunch length shortening after the first compressor.

In a seminal paper [21] the authors derive the energy loss per dipole due to the interaction of the CSR emitted by the back particles in a bunch with the particles that precede them along the bunch length for a longitudinally uniform charge distribution. The following equation provides an estimate for the CSR-induced total energy loss, which fits well the case of the FERMI beam in the first compressor area [22]:

$$
\Delta E_{\mathrm{CSR}} \approx \frac{1}{4 \pi \varepsilon_{0}} \frac{Q}{l_{b}}[4 \ln (\gamma \theta)-2]
$$

where $Q$ is the charge per bunch, $l_{b}$ is the full bunch length, $\theta$ is the bending angle and $\varepsilon_{0}$ is the vacuum permittivity. We derived in [23] an approximation for the relative emittance blowup, for a bunch compressor in which only the last dipole (where the bunch is shortest) gives a significant contribution to the emittance increase. In our case the bunch length is approximately constant through the two chicanes, and we have modified the formula as follows:

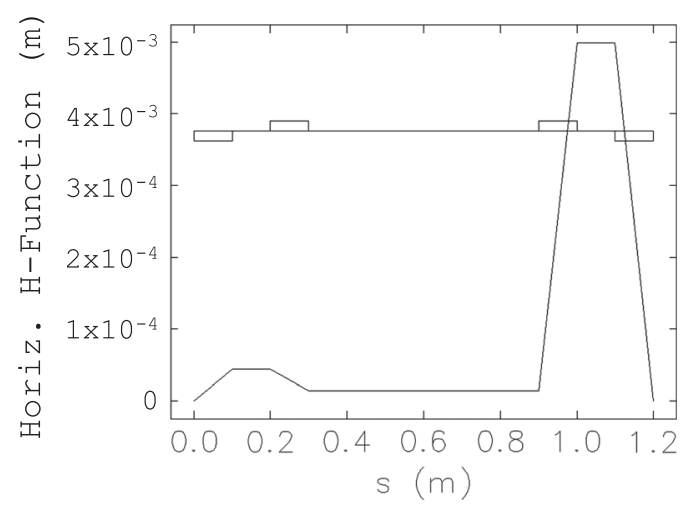

FIG. 8. Horizontal $H$ function in NIEM at $286 \mathrm{MeV}$.

$$
\frac{\Delta \varepsilon}{\varepsilon} \approx \frac{1}{4} \frac{\langle\hat{H}\rangle_{\mathrm{dip}}}{\varepsilon} \sigma_{\delta, \mathrm{CSR}}^{2}
$$

where $\Delta \varepsilon / \varepsilon$ is the relative emittance increase at the end of the line, $\sigma_{\delta, \mathrm{CSR}} \approx \frac{\Delta E_{\mathrm{CSR}}}{\sqrt{12} n_{b} E}$ [22] is the relative rms energy deviation in a dipole due to CSR, $E$ the average electron energy (in Joule), $n_{b}$ is the number of electrons in a bunch, $H=\frac{1+\alpha^{2}}{\beta} \eta^{2}+2 \alpha \eta \eta^{\prime}+\beta \eta^{\prime 2},\langle\hat{H}\rangle_{\text {dip }}$ is the sum, in quadrature, of the maximum values of $H$ in the dipoles, $\eta$ is the energy dispersion, $\eta^{\prime}=\frac{d \eta}{d s}$ and $\beta, \alpha$ are the Twiss parameters. The $H$ function in NIEM is shown in Fig. 8; its average value in the dipoles is about $7.5 \times 10^{-4} \mathrm{~m}$. Equations (1) and (2) contain rather broad approximations, and are only intended to be correct within an order of magnitude. They were used in our first attempt to define the NIEM geometry in order to minimize the CSR effect on the particles motion, and then to verify the ELEGANT computer results. Table II summarizes the beam parameters of interest and the theoretical expectations based on Eqs. (1) and (2). They support the ELEGANT results shown in Table II that CSR plays no role in the NIEM designs for both 100 and at $286 \mathrm{MeV}$.

\section{Bunch length}

The linear compression factor (ratio of the bunch lengths at the start and end of a line) is given by

$$
C=\frac{1}{1+h R_{56}}
$$

TABLE II. Beam parameters and CSR effect across NIEM.

\begin{tabular}{lcc}
\hline \hline Charge (pC) & 500 & 500 \\
Mean energy (MeV) & 100 & 286 \\
Bunch length, FW (ps) & 13.4 & 2.0 \\
$\sigma_{\delta, \text { CSR }}$ & $1.0 \times 10^{-5}$ & $5.0 \times 10^{-5}$ \\
$\Delta \varepsilon / \varepsilon(\%)$ & $<0.1$ & 0.1 \\
\hline \hline
\end{tabular}


where $h=\frac{d E}{E \cdot d z}$ is the linear chirp relative to the reference energy. As previously shown, in the achromat under consideration $R_{56}=-0.4 \mathrm{~mm}$. With $h \approx 0.02 / 1.6 \times 10^{-4}=$ $125 \mathrm{~m}^{-1}$ in the area of the first compressor, the bunch is compressed only by $\sim 5 \%$ and this is confirmed by ELEGANT tracking results both at 286 and at $100 \mathrm{MeV}$ (not shown).

\section{E. Beam size and angular divergence}

The beam angular divergence plays a fundamental role in determining the accuracy with which the NIEM "measures" the beam optical parameters. The dominant contribution of either the particles' betatron or chromatic motion to the beam size and angular divergence will allow the user to measure the beam geometric emittance and Twiss parameters in the former case, or the beam total energy spread in the latter. The bending magnets in NIEM increase the horizontal beam size and divergence in the presence of energy spread. This temporary broadening, which is maximum in between dipoles 1 (3) and 2 (4) for the divergence and in between dipoles 2 and 3 for the size, is canceled at the NIEM end, when the dispersion and its $\mathrm{s}$ derivative return to zero (see Fig. 6). Detailed behaviors of the beam size and angular spread across NIEM at $100 \mathrm{MeV}$ and at $286 \mathrm{MeV}$ are reported in Appendix A.

For a Gaussian particle distribution in the transverse phase space, the geometric and chromatic contributions to the beam horizontal rms angular divergence and size give, respectively, $\sigma_{x^{\prime}}=\sqrt{\varepsilon_{x} \gamma_{x}+\left(\eta_{x}^{\prime} \sigma_{\delta}\right)^{2}}$ and $\sigma_{x}=$ $\sqrt{\varepsilon_{x} \beta_{x}+\left(\eta_{x} \sigma_{\delta}\right)^{2}}$. Table III lists the geometric and the chromatic contributions to the rms beam angular divergence (respectivelyand) and to the rms beam size (respectively, $\sqrt{\varepsilon \gamma}$ and $\eta^{\prime} \sigma_{\delta}$ ), for the NIEM region at the entrance of dipole 1 (where the beam size will be measured); at the entrance and exit of dipole 2; and at the entrance of dipole 3. In Table III we distinguish between the betatron and the chromatic contributions. Comparison of these two allows us to recognize regions where one dominates the other. In the bending plane, the chromatic contribution to the angular divergence dominates over the betatron contribution in between dipoles 1 and 2 (see "Entrance of Dipole 2" in Table III). Thus we predict that the visibility of the far field OSR interferences produced in this region will be primarily sensitive to and can be used to measure the beam energy spread. Since the opposite is true in between dipoles 2 and 3 (see the column titled "Entrance of Dipole 3" in Table III), where the chromatic contribution to the angular divergence is zero, we predict that the OSRI visibility in this region can be used to determine the beam's betatron angular divergence. These results are tested and confirmed by our OSR code calculations (cf. Sec. VI).

\section{OSRI CODE CALCULATIONS}

\section{A. Description of the OSRI code}

An accelerated electron moving in vacuum radiates electromagnetic waves. If the electron changes the direction of velocity "instantly" then the intensity of the wave in the far zone (angular distribution) is described by the wellknown Bremsstrahlung formula for the spectral density (Fourier component of intensity distribution in the far zone). In the code the motion of the electron in the transverse magnetic field is treated as a series of small elemental "elastic collisions" - changes in the direction of the velocity. The whole trajectory is segmented into the elemental arcs, the radiation from each elemental arc is calculated as Bremsstrahlung and the total radiation is calculated as coherent sum of far zone "fields" of the elemental radiations. The trajectory and accordingly the distribution of intensity is a function of all parameters of the electron as well as of parameters of magnetic field and "initial" angle and initial coordinate of electron. Usually the initial coordinate is taken to be zero and placed

TABLE III. Beam rms size (in $\mu \mathrm{m}$ ) and angular divergence (in $\mu \mathrm{rad}$ ) across NIEM at 100 and $286 \mathrm{MeV}$. Top: horizontal (magnetic bending) plane; bottom: vertical plane. Longitudinal coordinate $s$ refers to Fig. 6 for $100 \mathrm{MeV}$ and to Fig. 7 for $286 \mathrm{MeV}$. Quantities in italics will be measured.

\begin{tabular}{|c|c|c|c|c|c|c|c|c|}
\hline \multirow[b]{2}{*}{ Energy } & $\begin{array}{l}\text { Entrance } \\
\text { Dipole 1 } \\
\end{array}$ & $\begin{array}{l}\text { Entrance } \\
\text { Dipole } 2\end{array}$ & $\begin{array}{c}\text { Exit } \\
\text { Dipole } 2\end{array}$ & $\begin{array}{l}\text { Entrance } \\
\text { Dipole } 3\end{array}$ & $\begin{array}{l}\text { Entrance } \\
\text { Dipole } 1\end{array}$ & $\begin{array}{l}\text { Entrance } \\
\text { Dipole } 2\end{array}$ & $\begin{array}{c}\text { Exit } \\
\text { Dipole } 2\end{array}$ & $\begin{array}{l}\text { Entrance } \\
\text { Dipole } 3\end{array}$ \\
\hline & \multicolumn{4}{|c|}{$100 \mathrm{MeV}$} & \multicolumn{4}{|c|}{$286 \mathrm{MeV}$} \\
\hline $\mathrm{s}[\mathrm{m}]$ & 0.0 & 0.2 & 0.3 & 0.9 & 0.0 & 0.2 & 0.3 & 0.9 \\
\hline$\sqrt{\varepsilon_{x} \beta_{x}}[\mu \mathrm{m}]$ & 75 & 75 & 85 & 95 & 25 & 30 & 35 & 75 \\
\hline$\eta_{x} \sigma_{\delta}[\mu \mathrm{m}]$ & 0 & 25 & 35 & 35 & 0 & 105 & 155 & 160 \\
\hline$\sqrt{\varepsilon_{x} \gamma_{x}}[\mu \mathrm{rad}]$ & 70 & 70 & 70 & 70 & 70 & 70 & 70 & 70 \\
\hline$\eta_{x}^{\prime} \sigma_{\delta}[\mu \mathrm{rad}]$ & 0 & 175 & 0 & 0 & 0 & 750 & 0 & 0 \\
\hline$\sqrt{\varepsilon_{y} \beta_{y}}[\mu \mathrm{m}]$ & 70 & 70 & 75 & 85 & 30 & 35 & 40 & 70 \\
\hline$\eta_{y} \sigma_{\delta}[\mu \mathrm{m}]$ & 0 & 0 & 0 & 0 & 0 & 0 & 0 & 0 \\
\hline$\sqrt{\varepsilon_{y} \gamma_{y}}[\mu \mathrm{rad}]$ & 75 & 75 & 75 & 75 & 70 & 70 & 70 & 70 \\
\hline$\eta_{y}^{\prime} \sigma_{\delta}[\mu \mathrm{rad}]$ & 0 & 0 & 0 & 0 & 0 & 0 & 0 & 0 \\
\hline
\end{tabular}


at the entrance of the first magnet. The initial angle- the angle of injection into the first magnet-is variable.

Convolutions of the intensity of radiation from the electrons are performed over energy, trajectory angle and wavelength. The distribution of energy is assumed to be represented by single Gaussian. In the case of angular spread we apply a two-dimensional Gaussian distribution to account for the projection of the trajectory angle of each electron in the horizontal and vertical planes. Finally, to account for the finite pass band filter used in our measurements, we perform a convolution with a rectangular distribution function of the frequency of radiation which is assumed to model the transmissivity of the optical bandpass filter. In the code each of the above convolutions can be applied individually only. Note that the convolution procedures assume that the electrons in a bunch radiate incoherently.

\section{B. OSRI patterns from individual electrons}

A normal light interferometer makes use of a coherent light source, e.g., a laser, and the light is forced to travel along two different optical paths to form an interference pattern. It is this path length difference that causes the interference. In an OTR, OSR or other type of relativistic charged particle radiation interferometer, each electron in the beam produces coherent radiation from structures encountered (e.g., bends or edges) along its trajectory and these radiations can interfere if they overlap (cf. Figs. 1 and 3). Hence each electron in the beam generates its own individual OSR interference pattern. In this case the relevant path length is the distance traveled by an individual electron between the two structures measured along its own trajectory. The radiations from different electrons traveling along different trajectories are incoherent and therefore cannot interfere in principle (microbunching at optical wavelengths is not considered in this discussion).

\section{Accounting for energy spread effects in NIEM}

To clearly understand the effect of energy spread let us assume for the moment that there is zero transverse betatron divergence. We then note that for OSRI generated from dipoles 1,2 there is a large dispersion of trajectory angles that is directly correlated with energy spread. We also note that there is an OSRI pattern produced by each electron with a particular energy and accordingly with a particular trajectory. This single pattern must be incoherently added to that of other OSRI patterns produced by electrons with different energies and their corresponding trajectory angles. The incoherent addition produces an ensemble OSRI pattern in which the fringes are smeared, i.e., there is a reduced visibility of the fringes in the ensemble OSRI pattern, in contrast to the OSRI pattern produced by a single electron. If this angular dispersion is larger than the betatron beam divergence, the visibility of the OSRI will be dominated by and can be used to measure the energy spread. In addition to this energy correlated angular dispersion, there is also a typically small broadening of the fringes due to relativistic kinematic effects which are due to differences in velocities of the electrons caused by their differences in energies. These will discussed in more detail below in Sec. VIE.

\section{Results of calculation of OSRI from magnets 1,2}

Figure 9 shows the effect of energy spread and divergence on the OSRI from dipoles 1,2 for a beam energy of $100 \mathrm{MeV}$. Note that only part of the total pattern (i.e., 15$35 \mathrm{mrad}$ ) is shown in the figure. However, this limited view is sufficient to see the effects of energy spread and divergence on the OSRI fringes. The relative effects of the energy spread and divergence on the OSRI from 1,2 are respectively shown by the red and blue scan curves. The effect of the energy spread clearly dominates over that introduced by the beam betatron divergence, thus confirming the ELEGANT predictions as noted in Sec. V. This will not always be the case, e.g., if the energy spread in the beam is very small. But it is the case for FERMI, i.e., for a $0.5 \%$ energy spread when the betatron divergence is in the range of about $40-100 \mu \mathrm{rad}$. Hence, the OSRI observed from 1,2 can be used as a diagnostic for the energy spread. Similar results are obtained for OSRI 1,2 at $286 \mathrm{MeV}$ (not shown).

\section{E. Results of OSRI from magnets 2,3}

We remarked above in the discussion of Fig. 3 that for the 2,3 interferometer OSR from only a small portion of the magnetic fields in each dipole, i.e., the trailing edge of dipole 2 and the leading edge of dipole 3, effectively interfere. For this reason it is necessary to have a longer

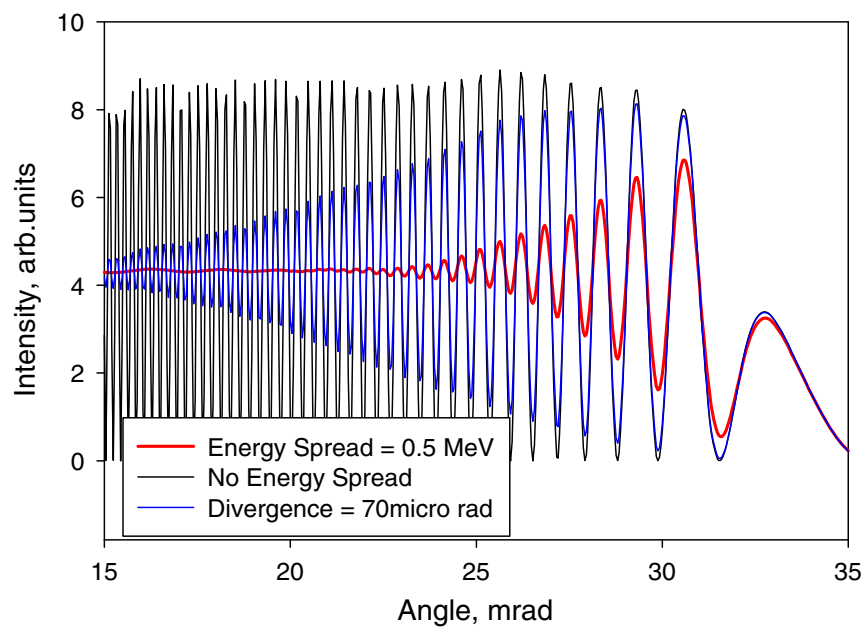

FIG. 9. OSRI from magnets $(1,2)$ and beam energy $E=100 \mathrm{MeV}$, showing how the fringes (black curve) are affected by energy spread (red curve) and divergence (blue curve). 


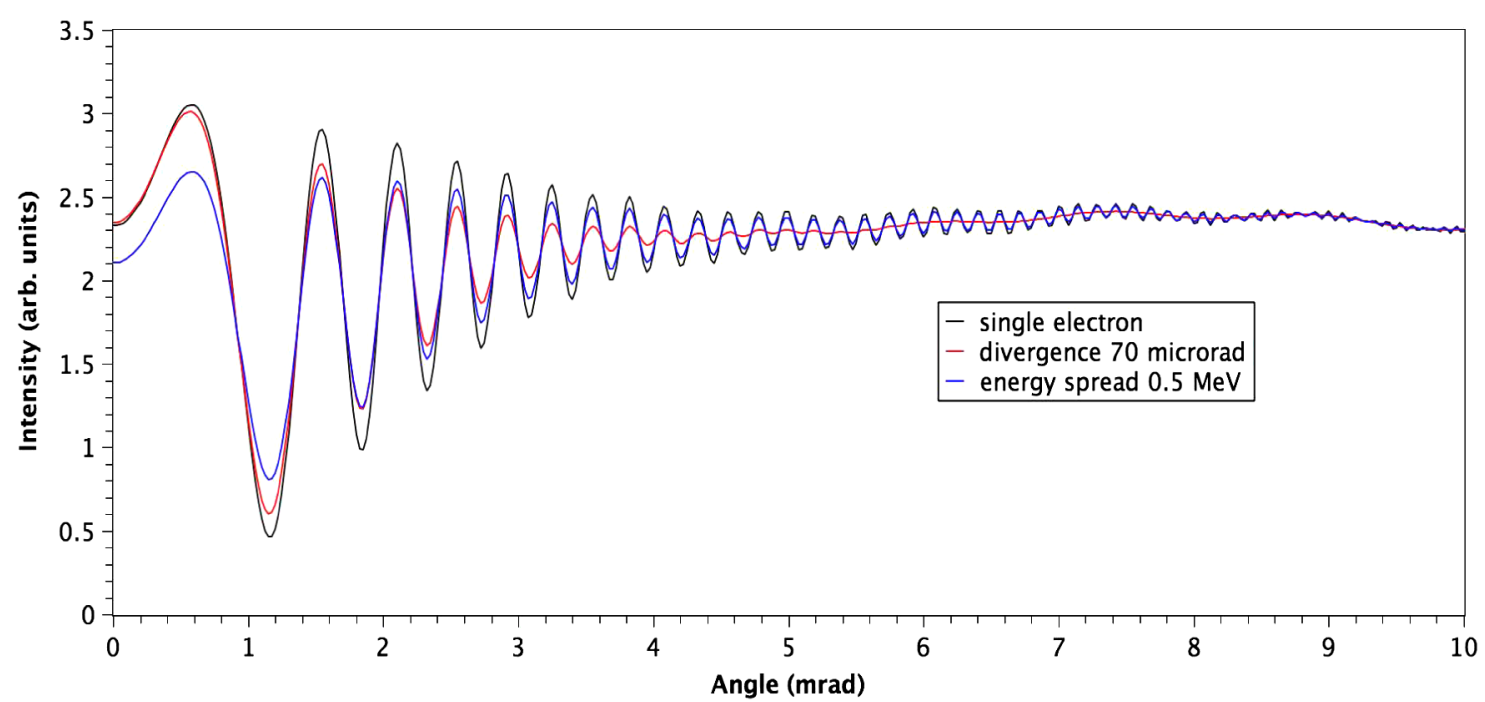

FIG. 10. OSR interferences from magnets $(2,3)$ and beam energy $E=100 \mathrm{MeV}$, showing the fringe visibility for a single electron and a beam with 0.5 energy spread; as well as a beam with divergence $70 \mu \mathrm{rad}$ (red).

straight section $(600 \mathrm{~mm})$ between 2,3 for both 100 and $286 \mathrm{MeV}$, in order to view a sufficient number of fringes (before their amplitudes fall too much with angle) to see the change in visibility due to divergence. Also, except for a very small region, i.e., near the edges of dipoles 2 and 3, there is no correlation between angle and energy spread, and the effect of energy spread on the OSRI from 2,3 is only due to a kinematic, relativistic effect, i.e., the velocity spread due to energy spread.

Figure 10 shows the single electron OSRI pattern (black), the OSRI 2,3 pattern from a beam with energy spread due to kinematic effects (blue) and a beam with $70 \mu \mathrm{rad}$ divergence (red); the beam mean energy is $100 \mathrm{MeV}$. It is apparent that the $70 \mu \mathrm{rad}$ divergence dominates the visibility beyond the third order. We conclude that the expected divergence can be measured using higher order fringes $(>3)$ of OSRI from magnets $(2,3)$ in the presence of the expected $0.5 \% \mathrm{rms}$ energy spread present at FERMI just after the laser heater (see Fig. 5). At the same time we see that the visibility of the first order fringe is dominated by energy spread. These same effects have previously been noted for OTRI [8] and the use of both lower and higher order fringes was proposed as a way to separate out the effects of divergence and energy spread. This same strategy can be applied to the OSRI from 2,3. Additionally, we can use energy spread measured from 1,2, where the former dominates the visibility for all order fringes as the basis for a two parameter fit of the OSRI data from 2,3 to increase the accuracy of the divergence measurement.

Figure 11 shows a two-dimensional OSRI pattern, generated by a single electron with energy $286 \mathrm{Mev}$ from magnets 2,3 . The horizontal axis represents the horizontal angle $X^{\prime}=[-15,15] \mathrm{mrad}$ (400 pix) and the vertical axis the vertical angle: $Y^{\prime}=[-6,6] \operatorname{mrad}(160$ pix). The center of the pattern has coordinates $X^{\prime}=0, Y^{\prime}=0$. Horizontal $\left(X^{\prime}\right)$ and vertical $\left(Y^{\prime}\right)$ line scans through the center of the OSRI pattern created by a beam with finite divergence can be used to separately measure the horizontal and vertical components as we will show below.

Figure 12 shows a horizontal line scan of the single electron two-dimension far field OSRI pattern, (black curve) along with those produced by beams with $2 \%$ energy spread (blue curve) and with $70 \mu \mathrm{rad}$ divergence (red curve). The former curve overlaps the single electron curve, especially at the higher fringe orders thus demonstrating that the OSRI from 2,3 is negligibly affected by this energy spread. To the contrary, Fig. 12 (red curve) shows that a beam angular spread of $70 \mu \mathrm{rad}$ clearly dominates the visibility of all the fringe orders and therefore this divergence can be readily measured from the OSRI 2,3 fringe visibility. To obtain higher accuracy in the measurement a two parameter (energy spread and divergence) fit can be done as described above for the $100 \mathrm{MeV}$ case.

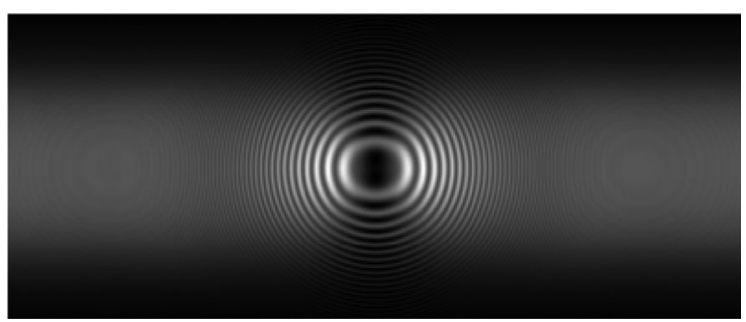

FIG. 11. Two-dimensional far field angular distribution OSRI formed by the interference of OSR from dipoles 2 and 3 by a single electron with energy $286 \mathrm{MeV}$. 


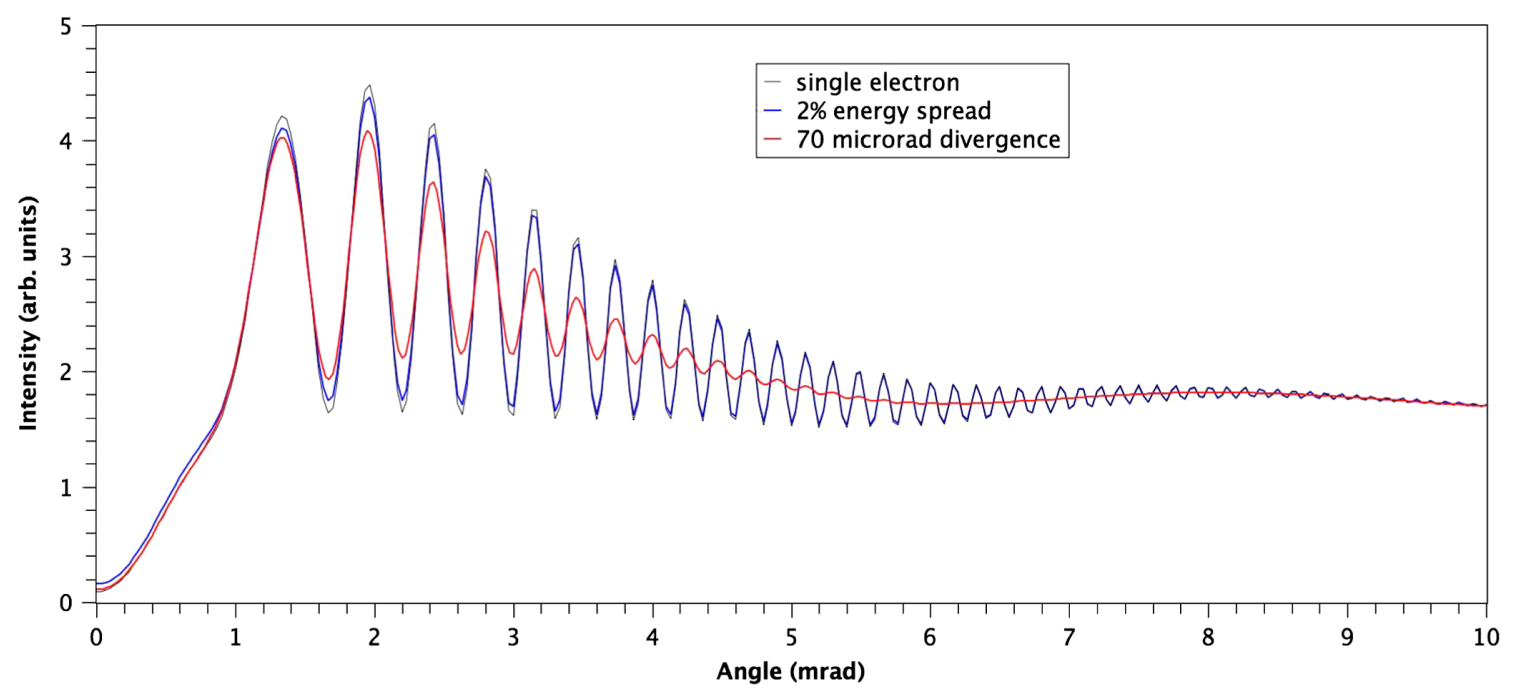

FIG. 12. OSR interferences from magnets $(2,3)$ and a beam energy of $286 \mathrm{MeV}$ showing the fringe visibility for single electron (black line); a beam with (a) $5.7 \mathrm{MeV}$ rms energy spread (blue line); and a beam with an angular divergence of $70 \mu \mathrm{rad}$ (red line).

\section{F. Vertical and horizontal divergence effects on OSRI}

Figure 13 shows horizontal line scans of the 2D OSRI pattern generated by a beam of electrons with horizontal divergence of $70 \mu \mathrm{rad}$ for various values of vertical divergence, along with a single electron (zero horizontal divergence) scan for a beam energy of $286 \mathrm{MeV}$, as an example. This figure indicates that there is negligible coupling between the horizontal and vertical divergences so that a horizontal scan can be used to measure the horizontal divergence by fitting the measured scan to theoretically generated ones. Similar results are obtained for $100 \mathrm{MeV}$. Similarly, Fig. 14 shows that the vertical scans of the OSRI pattern are independent of the horizontal divergence and thus can be used to measure the vertical component of the divergence.

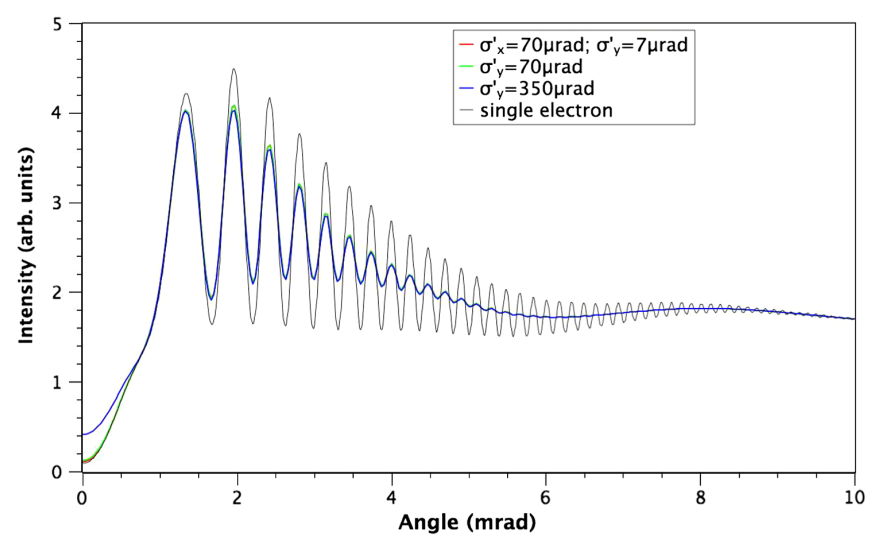

FIG. 13. Horizontal scan of OSRI from dipoles 2,3 for various values of horizontal $(x)$ and vertical $(y)$ divergences, along with single electron (zero divergence) scan $(E=286 \mathrm{MeV})$. The two curves corresponding to horizontal angular divergence of $70 \mu \mathrm{rad}$ (red and green) superimpose.

\section{DATA ACQUISITION CONSIDERATIONS}

\section{A. Absolute yield of OSR and far field camera integration times}

Our code calculates the spectral-angular intensity distribution of OSR in terms of the dimensionless quantity $J_{\text {otr }}$, which is the distribution of OSR measured in terms of "OTR units," i.e., the intensity of OTR at the angle of peak emission $\left(\theta_{p}^{\mathrm{OTR}}=1 / \gamma\right)$, which is $\frac{e^{2}}{\pi^{2} c} \cdot \frac{\gamma^{2}}{4}[7,8]$. This is an arbitrary choice of normalization, but it is one that we have historically used and allows us to conveniently compare the yields of the two types of radiation. In these units the number of OSR photons radiated by a single electron in the frequency interval $d \omega$ into the solid angle $d \Omega$ is given by

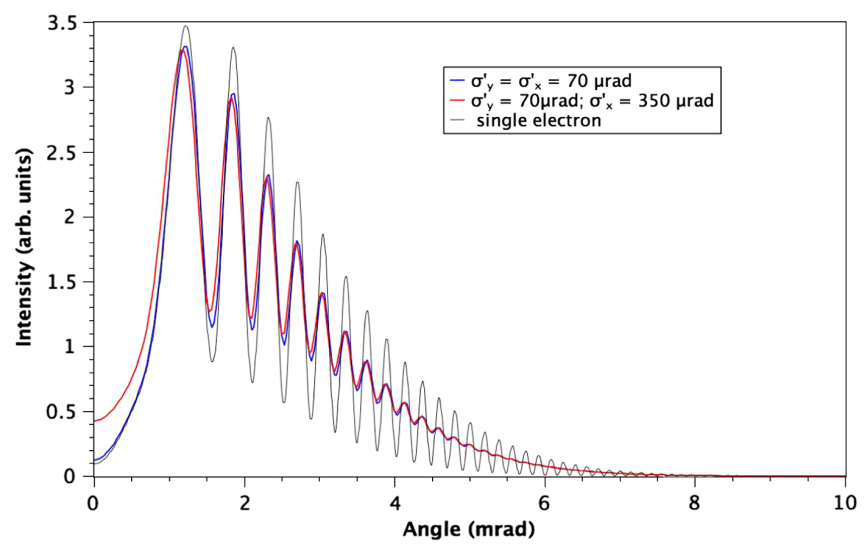

FIG. 14. Vertical scan of OSRI from dipoles 2,3 for various values of horizontal $(x)$ and vertical $(y)$ divergences along with single electron scan $(E=286 \mathrm{MeV})$. 


$$
\begin{aligned}
d N_{\mathrm{ph}} & =\frac{e^{2}}{\pi^{2} c \hbar \omega} \frac{\gamma^{2}}{4} J_{\mathrm{otr}} d \Omega d \omega \\
& =7.38 \times 10^{-4} \frac{d \omega}{\omega} \frac{\gamma^{2}}{4} J_{\mathrm{otr}} d \Omega
\end{aligned}
$$

In order to measure the expected beam divergence, a high order of OSRI fringes must be resolved by the sensor of the far field camera that is used to observe the OSRI pattern. For example, in the case of magnetic bends 2 and 3 and energy $286 \mathrm{MeV}$, the angular size of the 15th fringe is about $0.2 \mathrm{mrad}$ (see Fig. 13) and our OSR code gives $J_{\text {otr }}=$ 2 at the angle where this fringe occurs. In order to well define this fringe, about five pixels are needed, i.e., an angular resolution $\sim 0.04 \mathrm{mrad}$ per pixel. This can be achieved by proper choice of the focal length of the camera lens, the sensor size and number of pixels. Then, if we assume a single bunch charge $Q=500 \mathrm{pC}$, a wavelength of observation $\lambda=630 \mathrm{~nm}$ and a passband of $10 \mathrm{~nm}$, the number of photons observed per pixel from a single bunch is 9. Similarly, for OSRI produced from bends 2,3 at $100 \mathrm{MeV}: J_{\text {otr }}=2.2$ for fringe number 15 , the solid angle per pixel $=0.04 \times 0.04 \mathrm{mrad}^{2}$ and the number of photons is 1. From our experimental experience with OTRI [7] using cooled CCD cameras with high sensitivity, low dark current and large full well capacity [5-7] good signal requires $\sim 1000$ photons. Then at a pulse repetition rate of $50 \mathrm{~Hz}$, the integration times to accumulate the required number of photons per pixel are $2 \mathrm{sec}(286 \mathrm{MeV})$ and $20 \mathrm{sec}(100 \mathrm{MeV})$. After these integration times, postprocessing of the OSR signals can provide the Twiss parameters and beam emittances, as we show below.

\section{B. Beam size considerations}

In addition to the rms divergence that is obtained from OSRI, in order to determine the rms emittance, we also require a measurement of the rms beam size from the OSR radiation. The beam size is typically characterized by its rms value, $\sigma_{\mathrm{b}}$, and the beam profile is approximated by a normal distribution function, i.e., a Gaussian function. The optical resolution which limits the beam size measurement is the PSF, which for OSR can be defined as the distribution of the OSR produced by a single electron as accepted by the imaging optics. Assuming that the PSF is also approximated by a Gaussian, we can characterize the PSF in the same terms as the beam size, i.e., by its rms value, $\sigma_{\mathrm{PSF}}$. If the measured OSR distribution produced by the entire beam is similarly characterized and has a rms value $\sigma_{\mathrm{m}}$, then $\sigma_{b}^{2}=\sigma_{m}^{2}-\sigma_{\mathrm{PSF}}^{2}$.

A simple estimate of the rms value of the PSF can be made by assuming that the vertical and horizontal distributions of the OSR are about equal, which is not absolutely necessary but can be experimentally achieved by the use of a horizontal aperture, and the radiated field distribution can be described by a Gaussian with rms value $0.6 / \gamma$. The above estimate is maximal and is based on the facts that: (a) the rms of the PSF becomes larger the smaller the angular size of the radiation pattern, and (b) the smallest angular size of OSR is in the vertical direction. Then, applying the principles of Gaussian optics, the rms of the intensity distribution of the spot produced by electron, i.e., the PSF, is $\sigma_{\mathrm{PSF}} \approx 0.35 \gamma \lambda$. For example, for an electron with energy $100 \mathrm{MeV}$ and an observation wavelength $\lambda=630 \mathrm{~nm}, \sigma_{\mathrm{PSF}} \approx 43 \mu \mathrm{m}$. If the measured size of the OSR spot is significantly greater than this value, it is straightforward to estimate the rms beam size from a direct image of the beam at the entrance of the first dipole. However, if it is less than or comparable to $\sigma_{\mathrm{PSF}}$, the PSF will limit the measurement. The latter is the case for FERMI at $286 \mathrm{MeV}$ after BC1 where the ELEGANT simulations (cf. Table III) predict an rms beam size $\sim 25 \mu \mathrm{m}$. Therefore, for this situation at least it is clear that imaging the beam with standard optics is problematic and another technique, e.g., the Young's double slit method described above must be used to determine the rms beam size.

\section{ON-LINE ELECTRON BEAM OPTICS MONITORING AND FEEDBACK}

It was shown in the previous sections that in the energy range of $\sim 100-300 \mathrm{MeV}$ the electron beam size and the betratron angular divergence, both in and perpendicular to the bending plane of the NIEM, can be simultaneously monitored on-line, leaving the electron beam parameters substantially unaltered. As stated above, the product of the measured beam size $\sigma=\sqrt{\epsilon \beta}$ and divergence $\sigma^{\prime}=\sqrt{\epsilon \gamma}$ provides the rms geometric emittance $\epsilon$ of the electron beam at the entrance of the NIEM, when a beam waist is achieved there by matching the beam with upstream quadrupole magnets. Having a beam waist ensures the following relationship for the Twiss parameters in the plane of interest: $\gamma=1 / \beta$. The beam waist at the entrance of the NIEM is therefore part of the operational setup of the NIEM in order to measure on-line the beam emittance. We notice that, while the beam size is measured by means of the OSR emitted right at the entrance of first dipole magnet of the NIEM chicane, the beam angular divergence is measured from the OSRI pattern of electrons emitting in the middle of the chicane (i.e., from dipoles 2,3). The rectangular dipole magnets used for the NIEM provide a weak vertical focusing. By virtue of the small bending angle, this focusing adds only a submicron angular divergence to the particles' motion, which is negligible with respect to the $70 \mu \mathrm{rad}$ beam angular divergence that we plan to measure (see Table III). The NIEM is therefore transparent to the particle betatron motion.

In general, at the beginning of the machine operation, there is no assurance that the beam is matched to the design optics, i.e., with a waist at the NIEM's entrance. In this generic case, we have, apparently, three independent unknowns $(\epsilon, \beta$ and $\alpha$ ) and two independent equations $\left(\sigma=\sqrt{\epsilon \beta}\right.$ and $\left.\sigma^{\prime}=\sqrt{\epsilon \gamma}\right)$. In practice, however, we can think of repeating the measurement with NIEM for two 
different focal lengths of an upstream quadrupole magnet following the method described in [24] or by doing a traditional quadrupole scan using the OSR from dipole 1. The two methods are off-line techniques, since they require a change in the quad focusing strength to perform the emittance measurement. However, they can be used initially to establish the Twiss parameters and thus serve as the benchmark for an optical feedback loop to maintain the beam brightness. By doing this, a complete set of parameters, $\epsilon, \beta$ and $\alpha$ is measured at the entrance of the quadrupole magnet and can be back-tracked to any arbitrary upstream location (hereafter, the "starting point") on the basis of the actual machine settings. As a next step, the strength of quadrupole magnets between the starting point and the NIEM (hereafter, "matching section") can be optimized to match the beam to the design optics [19]. The proposed scheme for beam optics measurement and correction is sketched in Fig. 15. We remark that both in the $\mathrm{LH}$ and in the $\mathrm{BC} 1$ regions at FERMI there is a matching section upstream of the proposed positions of NIEM to prepare the beam for on-line diagnostics and FEL production. After the matching section, all the aforementioned optical parameters will be surveyed on-line through the NIEM with no further need either to change the
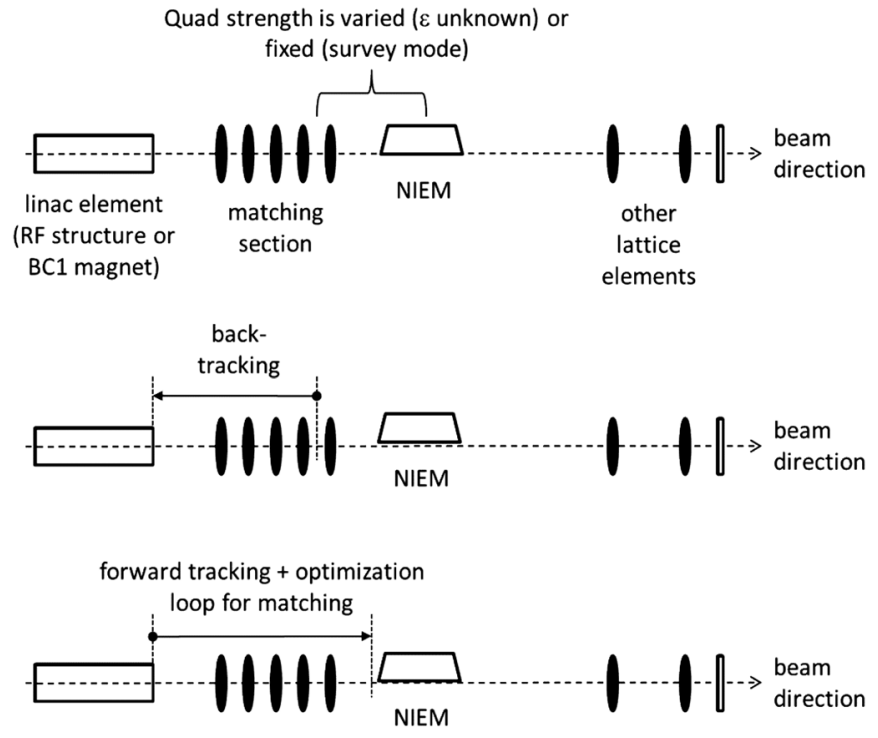

FIG. 15. Illustration of the optics matching loop with NIEM. From top to bottom: (i) the beam Twiss parameters are measured with the NIEM. If the emittance is not known initially and/or the beam waist condition is not satisfied, the strength of a quadrupole upstream NIEM is varied to reconstruct the whole ensemble of beam optical parameters. If the beam waist condition is ensured, instead, NIEM provides the emittance and the Twiss parameters at its entrance. (ii) The actual machine configuration is read, e.g., by the ELEGANT code and the measured Twiss parameters are backtracked to a point upstream of the matching section. (iii) Starting from the present machine configuration, ELEGANT optimizes the quadrupole strengths to match the beam Twiss parameters to the design values. quadrupoles' focusing strength or to insert screen targets along the beam path.

Let us now assume that during the machine run there is a slow change of the Twiss parameters and/or the emittance of the beam, due to, e.g., drifts of the beam characteristics and the accelerator settings upstream of the NIEM. In this case, the user will observe a variation of at least one of the two independently measured parameters, $\sigma$ and/or $\sigma^{\prime}$, so that their product no longer provides the true rms emittance, i.e., the waist condition at the entrance of the NIEM is lost. In practice, the user could envisage putting a tolerance on the variation of either $\sigma$ or $\sigma^{\prime}$ for the machine operation. Once the tolerance is exceeded, a new measurement of the beam optical parameters is required: the back-tracking and matching loop depicted above can be used to restore the design Twiss parameters (i.e., the beam waist) at the entrance of the NIEM. It is worth noticing that if the optics change at the NIEM location during the measurement is small enough, thereby not to affect substantially the beam quality at the end of the accelerator, the entire survey, measurement and correction loop would remain compatible with the machine operation. Thus, an optics feedback becomes practical.

The sensitivity of the measurements to few microns in beam size and tens of microrad in angular divergence, is expected to limit the accuracy of the normalized emittance measurement to the 0.1-0.3 micron rad level in the 100$300 \mathrm{MeV}$ beam energy range. With a normalized emittance of the order of 1 micron, we thus expect to be able to detect a variation of $\alpha$ by $\sim 0.3$ and a variation of $\beta$ by $\sim 0.2 \mathrm{~m}$. In the previous section, we showed that the need to obtain a significant signal from the OSRI pattern in order to extract the beam divergence slows down the feedback system to tens of seconds. This implies that the jitter of the beam position at the entrance of dipole 1 and the jitter of the beam angular divergence at the entrance of dipole 2 should not exceed, respectively, the design value of the rms beam size and beam angular divergence at the same locations. For FERMI, the tightest tolerance for the trajectory jitter on a few-tens-of-seconds time scale is of $\sim 10 \mu \mathrm{m}$ in $x, y$ coordinates of the bunch centroid, and $\sim 70 \mu \mathrm{rad}$ for its $x^{\prime}, y^{\prime}$ coordinate. Such requirements on the beam trajectory stability can be typically met in high brightness linacs and are in fact satisfied in FERMI.

In conclusion, we propose to adopt the NIEM not only as an on-line, noninvasive diagnostics for the electron beam optical parameters, but also as the main ingredient of a beam optics feedback system applied to accommodate a variation of the beam matching condition and the emittance. A combination of NIEM measurements and a change of upstream quadrupoles' strength is required to recover the nominal matching condition.

\section{CONCLUSIONS}

We have evaluated a design for a noninterceptive emittance and energy spread diagnostic chicane (NIEM) 

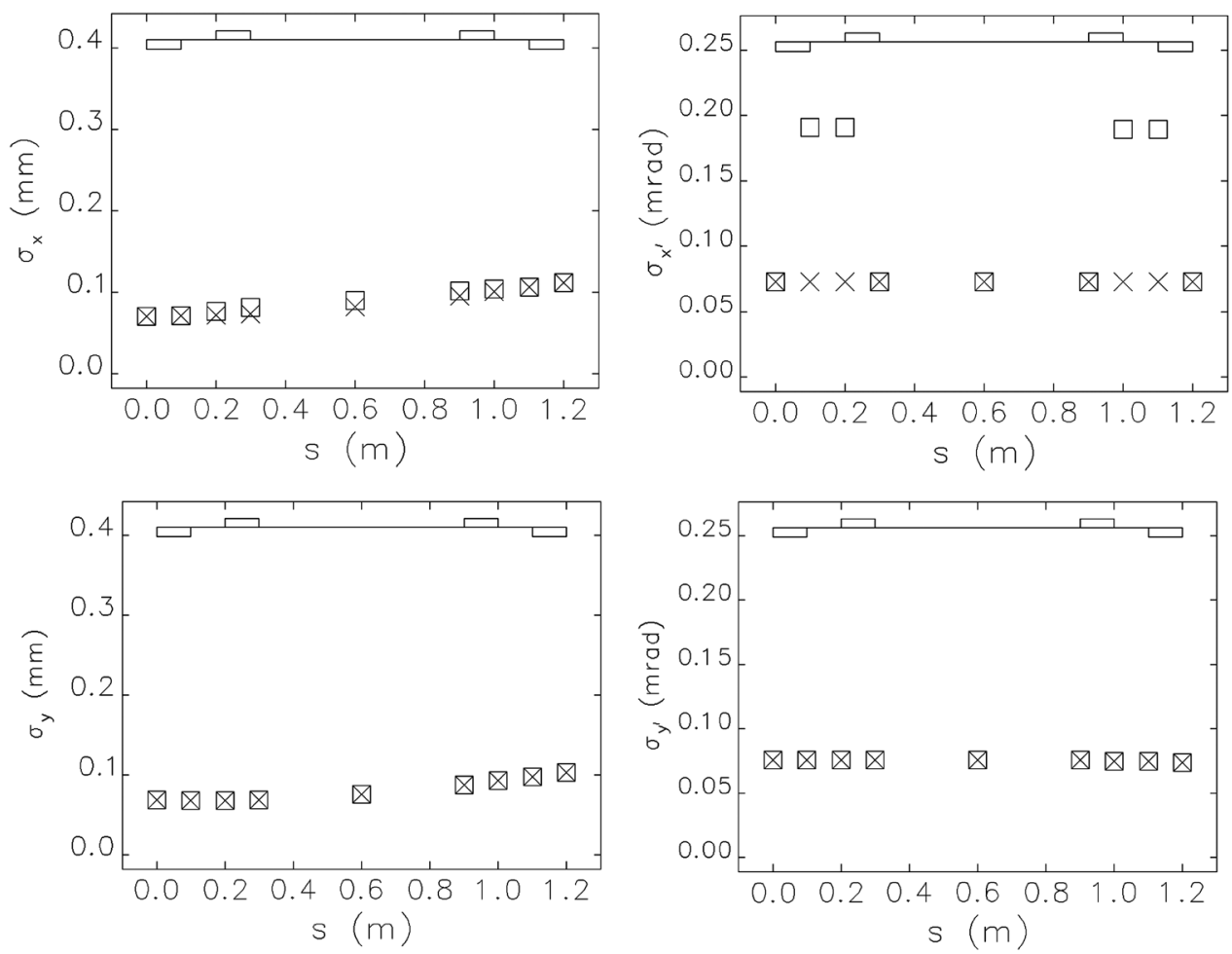

FIG. 16. Top left: rms horizontal beam size along NIEM with (squares) and without (crosses) energy spread. Top right: rms horizontal angular spread in mrad along NIEM with (squares) and without (crosses) energy spread. Bottom left: rms vertical beam size. Bottom right: rms vertical angular spread.

to measure properties of typical high brightness electron beams that have an rms normalized emittance $\sim 1 \mu \mathrm{m}$. As an example of the diagnostic capability and utility of such a device for high brightness accelerators, we have designed and evaluated a NIEM diagnostic for use at the FERMI FEL. Our OSR codes show that this device can easily measure the expected beam divergences, emittances and energy spreads typically produced at two points in the FERMI beam line, i.e., at $100 \mathrm{MeV}$ and at $286 \mathrm{MeV}$. ELEGANT and WARP simulations additionally show that introduction of the NIEM chicane into the beam line leads to negligible emittance blowup and bunch compression due to space charge, CSR and energy spread. The study of beam dynamics in NIEM is reassuring. The proposed achromat is immune to any perturbing effects, transverse or longitudinal. NIEM is therefore well matched to the task of monitoring the emittance without any visible effect on the beam. Furthermore, the diagnostic measurements of Twiss parameters via NIEM will thus allow, for the first time, online beam optics matching and feedback.

\section{ACKNOWLEDGMENTS}

This work was partially funded by the DOD Office of Naval Research and the Joint Technology Office (U.S.A.) and partially funded by the FERMI project of Elettra Sincrotrone Trieste, supported by the Ministry of
University and Research under Grants No. FIRBRBAP045JF2 and No. FIRB-RBAP06AWK3.

\section{APPENDIX A: ELECTRON BEAM SIZE AND ANGULAR DIVERGENCE THROUGH THE NIEM CHICANE}

Looking inside the chicane, the horizontal beam size and angular spread increase due to the energy dispersion and the energy spread in the bunch. This is shown in Fig. 16 for a $100 \mathrm{MeV}$ beam mean energy with a $0.5 \%$ fractional rms energy spread, and in Fig. 17 for a $286 \mathrm{MeV}$ beam with $2 \%$ fractional rms energy spread. The symbols point at these quantities at the entrance and exit of the bending magnets.

\section{APPENDIX B: SIMPLIFIED ANALYSIS OF THE EFFECT OF ENERGY SPREAD AND DIVERGENCE ON OSR INTERFERENCES}

The observed interference fringes produced by two independent sources of any type of radiation moving along with the electron, i.e., OSR, OTR or any other beam related radiation, are the result of the phase differences between the photons generated at the two sources-this is independent of the nature of the radiation mechanism. For example, the interferences can be generated from radiation from pairs of points along a curved electron trajectory (OSR) or from two separated foils transited by the electron (OTR). This means 

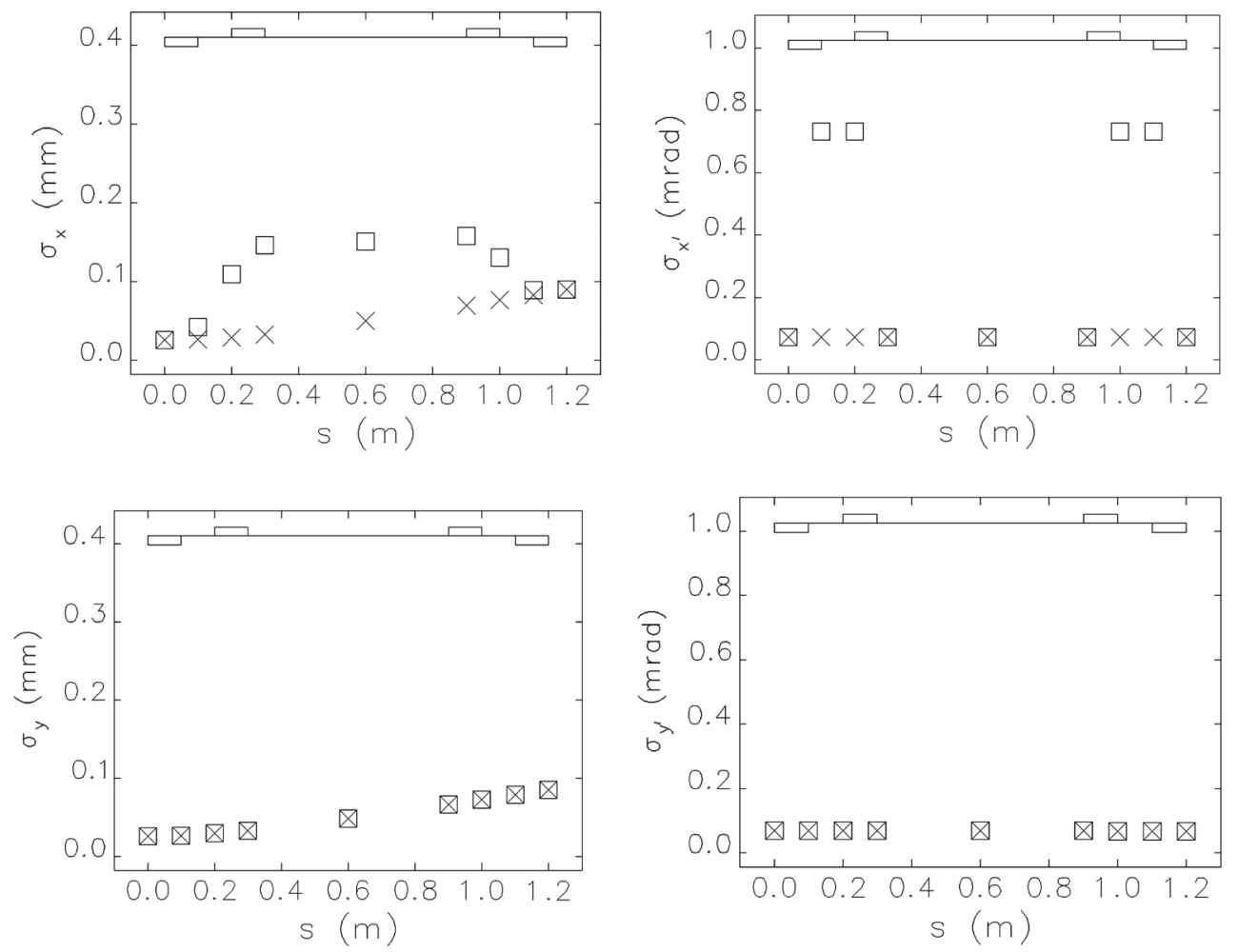

FIG. 17. Top left: rms horizontal beam size along NIEM with (squares) and without (crosses) energy spread. Top right: rms horizontal angular spread in mrad along NIEM with (squares) and without (crosses) energy spread. Bottom left: rms vertical beam size. Bottom right: rms vertical angular spread.

that the formalism developed to describe the effect of beam divergence and energy spread on the fringe visibility of OTR interferences (OTRI) can be applied to roughly analyze the features of the interference of OSR. However, one should keep in mind that the envelop of the fringe patterns for the two types of radiation will be different since the far field angular distribution pattern of each type of radiation in general is different. This difference becomes smaller and the analysis of the interferences becomes more exact as the observation wavelength $\lambda \gg \lambda_{c}$, the critical wavelength for SR generation [4]. This is the edge radiation (ER) limit where the ER pattern is almost identical to that of transition radiation. In the situations considered in this paper $\lambda \approx \lambda_{c}$ so the analysis of OSRI via OTRI is not exact but as we shall see the approximation is still very useful.

The application of OTRI analysis to OSRI is restricted by a number of conditions: (1) the sources of OSR taken into account must be much shorter than the distance between the sources, e.g., the sources can be the short portions of the exit and the entrance of magnetic dipoles separated by a long straight trajectory between the dipoles; (2) the nature of the actual electron trajectory must be taken into account in the OTRI model. For example, in the case of an $\mathrm{S}$ bend as is the case for dipoles 1,2 in the chicane, electrons with different energies travel along different trajectories producing a linear energy-angle correlation. This means that the one-dimensional angular spread of the trajectories due to the energy spread must be taken into account explicitly when calculating the effect of energy spread on the OTRI fringe visibility. This is done by convolving the single electron OTRI pattern with a Gaussian distribution of angles whose spread (divergence) is calculated a priori from a knowledge of the energy spread and the magnetic field of the first dipole. Such an energy-angular correlated divergence effect is not needed in the case of $U$ configuration dipoles 2,3 where the two sources effectively interfere only near the "edges" of the entrance and exit dipoles (see Fig. 3). In this case there is negligible energy spread-trajectory angle correlation and the effect of energy spread is purely kinematic. This kinematic energy spread and transverse divergence are entirely independent and the only beam properties which can affect the visibility of the $U$ bend OSR interference fringes.

The effect of beam parameters on OTR interferences is straightforward to calculate [5-7] and does not require a simulation code such as is necessary to predict the exact OSRI pattern. The OTR calculations are far simpler and require only convolutions of the single electron OTR interference pattern from two foils, which is available analytically, with distributions of energy, angle and wavelength. These convolutions show how the radiation pattern is affected by beam energy spread, beam divergence and observed bandpass. Typically the distributions of these parameters are approximated by simple analytic functions 
such as Gaussians. For example, to compute the effect of an rms angular divergence on the OTR fringe pattern, one can assume that the OTR radiation pattern of each electron is centered on the direction of its trajectory and then perform the convolution of a Gaussian distribution of trajectory angles with the single electron OTR interference pattern. Note, in general, that the convolution procedure should take into account the two-dimensional nature of the angular distribution of the trajectory angles as well as the two dimensional radiation pattern. Thus, for example, two angular distributions which are functions of horizontal and vertical beam divergence, respectively, are required to analyze the OTRI fringe pattern.

We first show how OTRI analysis is used to calculate the effect of energy spread and inherent beam divergence on the interferences of OSR from an $\mathrm{S}$ bend configuration, i.e., dipoles 1,2 , for a beam energy of $100 \mathrm{MeV}$. The effect of the energy spread in this case is directly correlated to a divergence in the trajectories of the electrons. Calculation of the angular divergence due to this energy spread can be done knowing the angular dispersion of the first dipole magnet using the following equations for the gyrofrequency, radius of curvature and the angle of deflection of the electron in the magnetic field:

$$
\begin{aligned}
\omega_{c} & =\frac{e B}{m c \gamma} ; \quad R_{c}=\frac{V}{\omega_{c}}=\frac{\beta m c^{2} \gamma}{e B} ; \quad \Theta=\frac{L}{R_{c}} ; \\
\Rightarrow & \frac{\Delta \Theta}{\Theta}=-\frac{\Delta \gamma}{\gamma} \approx-\frac{\Delta E}{E} .
\end{aligned}
$$

Assuming an effective length of the dipole magnet $l=100 \mathrm{~mm}$, a constant magnetic field $B=0.35 \mathrm{~T}$ and a mean electron beam energy $E=100 \mathrm{MeV}$, the angular dispersion in the horizontal plane is $\Theta=35 \mathrm{mrad}$. Since the angular dispersion is proportional to $1 / \gamma$, an energy spread $\Delta E=0.5 \mathrm{MeV}$ will lead to an absolute value of the angular spread, $|\Delta \Theta|=0.18 \mathrm{mrad} \gg 0.07 \mathrm{mrad}$, the expected angular divergence of the beam. This energy-angle correlation is included in the OSRI calculations automatically as a change of the trajectory of electron associated with the change of the energy of electron. But in the OTRI model the energy correlated angular spread is not present directly. Thus, it is necessary to explicitly input it into the angular convolution calculation to compute the effect in the horizontal direction. The results are shown in Fig. 18, where the two OTR foils are taken to be separated by a distance $L=100 \mathrm{~mm}$, i.e., the same interdipole distance used in the OSRI simulation. These results should be compared with the OSRI results shown above in Fig. 9. Note that for the beam conditions considered in this paper the observation wavelength is close to the critical wavelength for SR. Thus, the envelopes of the OTR and OSR intensity patterns are different and thus we only pay attention to and compare the features of the fringes, i.e., the modulation depth and the angular periodicity.

The black curves in both Figs. 9 and 18 show the intensity of the interferences produced by a single electron where the depth of modulation is $100 \%$ in both the OTRI and OSRI models. In the case of OTRI the black curve also presents the results obtained by convolving a Gaussian distribution of energy with an rms energy spread of $0.5 \mathrm{MeV}$ with the single electron interference pattern. The reason for the resulting overlap of the fringe patterns produced by both a single electron and that produced by multiple energy electrons is that the change in fringe visibility due to the $0.5 \mathrm{MeV}$ energy spread is too small to be seen. Our calculations show that a significant reduction in the fringe visibility can only be seen if the energy spread is greater than or equal to $2 \mathrm{MeV}$. Comparing the black curves of Figs. 9 and 18, it is seen that the period

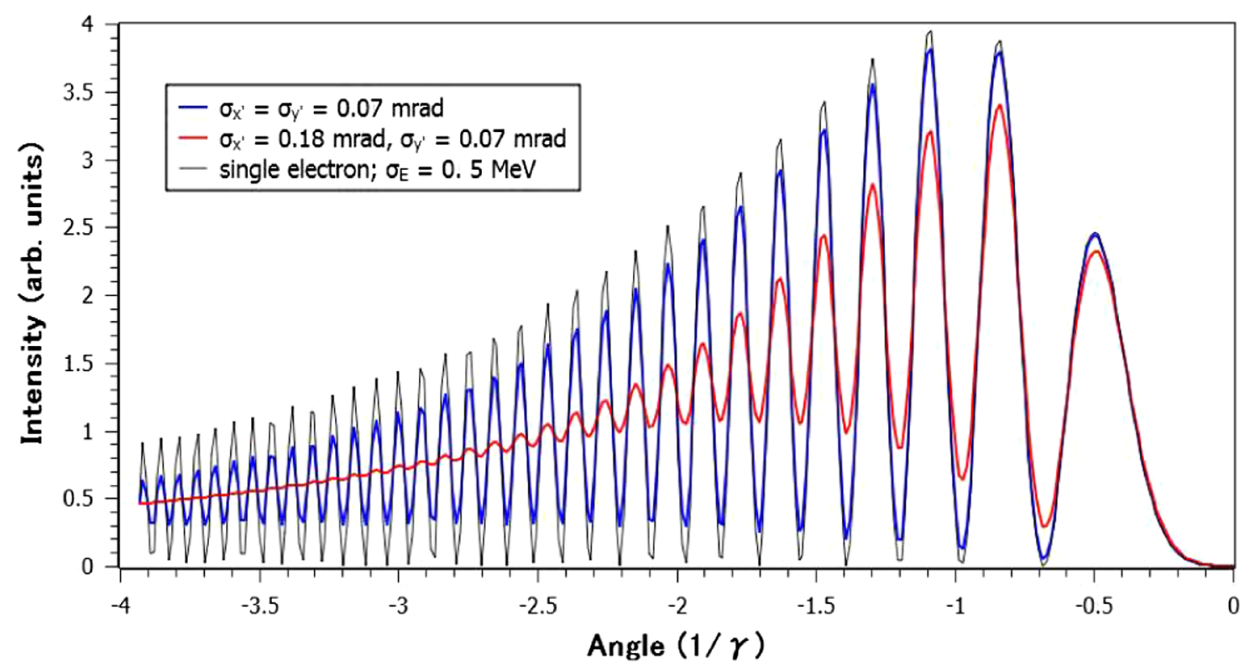

FIG. 18. Results of OTRI calculation of the effect of divergence and energy spread on interference fringes for two sources separated by distance $L=100 \mathrm{~mm}$; blue curve: angular divergences, $\sigma_{x^{\prime}}=\sigma_{y^{\prime}}=70 \mu \mathrm{rad}$; red curve: angular divergences $\sigma_{x^{\prime}}=180 \mu \mathrm{rad}$, $\sigma_{y^{\prime}}=70 \mu \mathrm{rad}$; black curve: single electron and beam of electrons with energy spread $\sigma_{E}=0.5 \mathrm{MeV}$. 


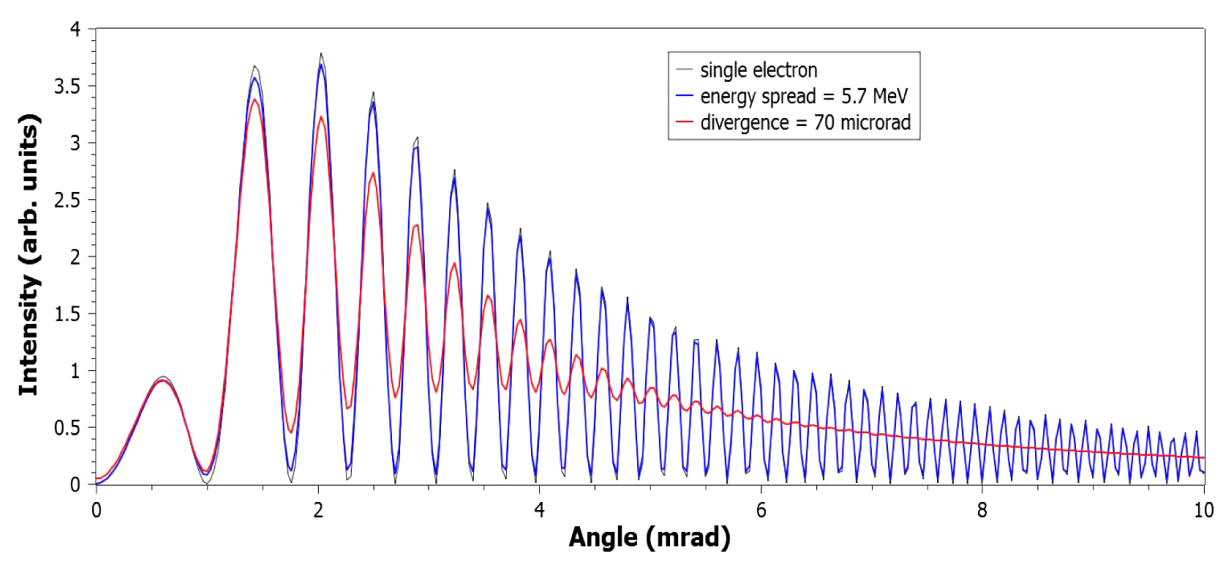

FIG. 19. OTR interferences from two sources separated by $L=600 \mathrm{~mm}$ for a beam with energy $286 \mathrm{MeV}$; (1) black curve: single electron; (2) blue curve: beam with $2 \%$ energy spread; (3) red curve: beam with angular spread (divergence) equal to $70 \mu \mathrm{rad}$.

of modulation is the same at small observation angles. At high observation angles the OSRI fringe period is smaller than the OTRI fringe period. This is because the distance between OSRI sources increases with increase of the observation angle. For example, at observation angle $\theta=0$ the distance between the sources is $L=100 \mathrm{~mm}$; at the angle $=15 \mathrm{mrad}$ the distance between the sources is $L=220 \mathrm{~mm}$. An increase of the distance between radiators leads to a decrease in the period of the interference fringes.

The reduced fringe visibility due to the effect of angular divergence is shown by the blue curves. The effect is almost the same in OSRI and OTRI calculations especially at small angle. The rms angular divergence in both the $x$ and $y$ directions is $0.07 \mathrm{mrad}$. One can see that the effect of divergence on the fringe visibility, i.e., the depth of modulation, is almost the same in both the OTRI and OSRI models. The red curve in Fig. 9 shows the effect of the large horizontal angular divergence $(0.18 \mathrm{mrad})$ produced by the first dipole magnet due to the energy spread $0.5 \mathrm{MeV}$ and in Fig. 18 produced by the same artificially introduced angular spread. Any observation of OSRI fringes between dipoles 1,2 will be dominated by this effective divergence and is clearly seen in the OTRI pattern as well.

Second, we analyze the OSRI from a U trajectory (magnets 2 and 3) using the OTRI mode. This is more straightforward than the S bend analysis given above, since there is no correlation between angle and energy as is the case with magnets 1,2 . For 2,3 the effect of energy spread is purely kinematic and is the same for OSRI as OTRI. Thus the effects of energy spread and divergence on the fringe visibility are practically equal in the OTRI model and OSRI simulation results. The OTRI results for a beam energy of $286 \mathrm{MeV}$ are shown in Fig. 19. These should be compared to the OSRI simulation code results shown in Fig. 12. Note that, unlike OTRI, the OSRI fringes do not modulate to zero [cf. the results shown in Fig. 3(c)]. Nevertheless, the relative visibility of the OTRI and OSRI is about the same for the same value of energy spread and divergence. This indicates even in this circumstance that the OTRI can still be used to estimate the visibility change of the OSRI pattern for given values of these quantities.

From the above comparisons we conclude that, to a good approximation, the effects of energy spread and divergence on OSRI for S and U bends in any chicane can be estimated by an analysis of OTRI fringes from two similarly separated sources. The latter requires simple numerical convolutions of known or estimated (e.g., Gaussian) distributions of energy and electron trajectory angle with the single electron two foil OTR interference pattern, which is known analytically.

[1] O. Chubar, Rev. Sci. Instrum. 66, 1872 (1995).

[2] N. Smolykov, Nucl. Instrum. Methods Phys. Res., Sect. B 448, 73 (2000).

[3] N. Smolykov, Nucl. Instrum. Methods Phys. Res., Sect. A 543, 51 (2005).

[4] A. Shkvarunets and R. Fiorito, Optical Synchrotron and Edge Radiation Diagnostics for Relativistic Electron Beams, AIP Conf. Proc. No. 732 (AIP, NewYork, 2004), pp. $429-436$.

[5] R. B. Fiorito and D. W. Rule, Optical Transition Radiation Beam Emittance Diagnostics, AIP Conf. Proc. No. 319 (AIP, NewYork, 1994), p. 21.

[6] R. B. Fiorito, A. G. Shkvarunets, T. Watanabe, V. Yakimenko, and D. Snyder, Phys. Rev. ST Accel. Beams 9, 052802 (2006).

[7] M. Holloway, R. B. Fiorito, A. G. Shkvarunets, S. V. Benson, D. Douglas, P. Evtushenko, and K. Jordan, Phys. Rev. ST Accel. Beams 11, 082801 (2008).

[8] R. Fiorito and A. Shkvarunets, in Proceedings of DIPACO3 (JACOW, Mainz, Germany, 2003), PM01.

[9] M. Borland, Advanced Photon Source Report No. LS-287, 2000. 
[10] A. Friedman, Nucl. Instrum. Methods Phys. Res., Sect. A 544, 160 (2005); http://hif.lbl.gov/theory/ WARP_summary.html.

[11] C. J. Bocchetta et al., FERMI@Elettra FEL Conceptual Design Report, 2007; http://www.elettra.trieste.it/FERMI.

[12] T. Naito and T. Mitsuhashi, Phys. Rev. ST Accel. Beams 9 , 122802 (2006).

[13] M. Boland, T. Mitsuhashi, and K. Wootton, in Proceedings of IBIC12 (JACOW, Tsukuba, Japan 2012), WECC03.

[14] E. Allaria et al., Nat. Photonics 6, 699 (2012).

[15] E. Allaria et al., Nat. Photonics 7, 913 (2013).

[16] E. L. Saldin, E. A. Schneidmiller, and M. Yurkov, Nucl. Instrum. Methods Phys. Res., Sect. A 528, 355 (2004).

[17] S. Spampinati, E. Allaria, L. Badano, S. Bassanese, D. Castronovo, M. B. Danailov, A. Demidovich, S. Di Mitri, B. Diviacco, W. M. Fawley, L. Giannessi, G. Penco, C. Spezzani, M. Trovò, G. De Ninno, and E. Ferrariet, in
Proceedings of the 34th International FEL Conf., Nara, Japan (JACOW, Japan, 2012), MOPD58.

[18] S. Di Mitri, Ph.D. thesis, Zernike Institute, University of Groningen, The Netherlands, 2011.

[19] S. Di Mitri, M. Cornacchia, C. Scafuri, and M. Sjöström, Phys. Rev. ST Accel. Beams 15, 012802 (2012).

[20] Z. Huang, M. Borland, P. Emma, J. Wu, C. Limborg, G. Stupakov, and J. Welch, Phys. Rev. ST Accel. Beams 7, 074401 (2004).

[21] E. L. Saldin, E. A. Schneidmiller, and M. V. Yurkov, Nucl. Instrum. Methods Phys. Res., Sect. A 398, 373 (1997).

[22] S. Di Mitri, E. M. Allaria, P. Craievich, W. Fawley, L. Giannessi, A. Lutman, G. Penco, S. Spampinati, and M. Trovo, Phys. Rev. ST Accel. Beams 15, 020701 (2012).

[23] S. Di Mitri and M. Cornacchia, Nucl. Instrum. Methods Phys. Res., Sect. A 735, 60 (2014).

[24] K. Poorrezaei, R. Fiorito, R. Kishek, and B. Beaudoin, Phys. Rev. ST Accel. Beams 16, 082801 (2013). 